\title{
Computing Global Characters
}

\author{
Jeffrey Adams
}

April 15, 2011

\section{Introduction}

Let $\pi$ be an irreducible representation of a real reductive group $G$. The global character $\theta_{\pi}$ of $\pi$ may be considered as a function on the regular semisimple elements of $G$. The global character determines $\pi$, and it is of great interest to compute it. For example Harish-Chandra found the discrete series representations of $G$ by computing their characters.

Fix a Cartan subgroup $H$ of $G$ and let $D$ be the Weyl denominator. Let $\lambda$ be the infinitesimal character of $\pi$. The function $\theta_{\pi}$ restricted to the regular elements of $H$ is roughly of the form

$$
\theta_{\pi}=\frac{\sum_{w \in W} a_{w}(\pi) e^{w \lambda}}{D}
$$

for certain integers $a_{w}(\pi)$. We would like to compute these integers.

There are several methods for doing this in the literature. The character of any irreducible representation is an integer combination of characters of standard modules, i.e., induced from (limits of) discrete series representations. Together with the induced character formula this reduces the problem in principle to computing characters of the discrete series.

The most conceptual algorithm for this case is due to Rebecca Herb [11]. It is based on the theory of endoscopy. First one computes the character of a stable sum of discrete series representations. Then one computes the character of an individual discrete series representation in terms of the characters of stable sums of discrete series for $G$ and for various endoscopic groups of lower dimension.

Properties of the discrete series characters, including the Hecht-Schmid character identities, give recursive formulas which determine these constants 
$[15$, Chapter $13, \S 4]$. In [14] Hirai uses this approach to give a very different formula from that of Herb.

Another very different formula is [6]. Other special cases include [17], [18], [8], [16], [19], [5], and unpublished work of Zuckerman. Also see the references in [11] and [14] and [15, Chaper 13].

We follow a more computational approach, based on the Kazhdan-LusztigVogan (KLV) polynomials. Certain terms $e^{w \lambda}$ only appear with nonzero coefficient in the character formula for a single standard module $I$. It follows that for any irreducible representation $\pi, a_{w}(\pi)= \pm M(I, \pi)$, where $M(I, \pi)$ is the coefficient of $I$ in the expression of $\pi$ as a sum of standard modules, which is given by a KLV polynomial. We then compute other coefficients $a_{w}(\pi)$ by using coherent continuation. The KLV polynomials are computed by the atlas software (available at www.liegroups.org). A version of this approach appears in an unpublished manuscript of Vogan [20].

The main ideas here are all present, in one form or another, in the literature, especially [10], as well as [20]. The main point here is to formulate the result in as self-contained and clean a form as possible, with an emphasis on the KLV polynomials for use in computations. These calculations will eventually be incorporated in the atlas software, which is a primary motivation for this paper.

One noteworthy aspect of this presentation is our use of covers of Cartan subgroups, which simplifies many of the formulas. In particular we give an elementary proof of the Zuckerman character formula. In these terms we write the trivial representation as a linear combination of standard modules parametrized by holomorphic characters of (covers of) Cartan subgroups.

An excellent reference for the basics of character theory is [10]. Also see [15], especially Chapters 10-13. We have tried to keep the presentation as elementary as possible. Everything is based on Harish-Chandra's theory, and most of what is required was available in the early 1980s. A notable exception is the theory of $\mathcal{D}$-modules, which are not discussed explicitly here, but which play an important role behind the scenes, and a fundamental one in the theory of the KLV polynomials.

We thank David Vogan for some helpful discussions, and Dragan Miličić and Becky Herb for assistance with Section 9.

It is a pleasure to dedicate this paper to my advisor, Gregg Zuckerman. 


\section{Weyl denominators and related functions}

Fix a connected, complex reductive group $G$ and a Cartan subgroup $H$. Let $\Delta=\Delta(G, H)$ be the set of roots of $H$ in $G$, and let $W=W(G, H)=W(\Delta)$ be the Weyl group. If $\Delta^{+}$is a set of positive roots, let $\rho=\rho\left(\Delta^{+}\right)=\frac{1}{2} \sum_{\Delta^{+}} \alpha$ as usual. The $\rho$-cover of $H$ is defined as in [4, Section 5]:

$$
H_{\rho}=\left\{(h, z) \in H \times \mathbb{C}^{*} \mid e^{2 \rho}(h)=z^{2}\right\} .
$$

The character $(h, z) \rightarrow z$ is denoted $e^{\rho}$; it is a genuine character of $H_{\rho}$ (one not factoring to $H$ ) satisfying $e^{\rho}(h, z)^{2}=e^{2 \rho}(h)$. If $w \Delta^{+}$is another choice of positive roots then $H_{\rho}$ is canonically isomorphic to $H_{w \rho}$, via the map $(h, z) \rightarrow\left(h, e^{w \rho-\rho}(h) z\right)$. It is convenient to eliminate the dependence on $\Delta^{+}$: define $\widetilde{H}$ to be the inverse limit of $\left\{H_{w \rho} \mid w \in W\right\}$.

The Weyl group acts on $H_{\rho}$ by $w:(h, z) \rightarrow\left(w h, e^{w^{-1} \rho-\rho}(h) z\right)$, and hence on $\widetilde{H}$.

Now assume $H$ is defined over $\mathbb{R}$, with real points $H(\mathbb{R})$. Let $H(\mathbb{R})_{\rho}$ be the inverse image of $H(\mathbb{R})$ in $H_{\rho}$, and define $\widetilde{H(\mathbb{R})}$ to be the inverse limit of the $H(\mathbb{R})_{w \rho}$.

Let $\mathfrak{h}$ be the Lie algebra of $H, \mathfrak{h}^{*}=\operatorname{Hom}_{\mathbb{C}}(\mathfrak{h}, \mathbb{C})$, and write $\langle$,$\rangle for the$ pairing $\mathfrak{h}^{*} \times \mathfrak{h} \rightarrow \mathbb{C}$. If $\alpha \in \Delta \subset \mathfrak{h}^{*}, \alpha^{\vee} \in \mathfrak{h}$ denotes the corresponding coroot.

The cross action on characters is defined as follows. For $\lambda \in \mathfrak{h}^{*}$ let $W(\lambda)=\{w \in W \mid w \lambda-\lambda$ is a sum of roots $\}$. For $\Lambda$ a genuine character of $H(\mathbb{R})_{\rho}, w \in W(d \Lambda)$, define

$$
w \times \Lambda=\Lambda \otimes(w d \Lambda-d \Lambda) .
$$

The expression $w d \Lambda-d \Lambda$ is a sum of roots, which we consider as a (nongenuine) character of $H(\mathbb{R})_{\rho}$; the right hand side is naturally a genuine character of $H(\mathbb{R})_{\rho}$.

This definition of the cross action is simpler than that of $[21$, Definition 8.3.1], thanks to the fact that we are using the $\rho$-cover of $H$.

Now suppose $\theta$ is a Cartan involution of $G$ corresponding to $G(\mathbb{R})$, i.e. $G(\mathbb{R})^{\theta}$ is a maximal compact subgroup of $G(\mathbb{R})$. For $H$ a $\theta$-stable Cartan subgroup set

$$
\begin{aligned}
\Delta_{i} & =\{\alpha \in \Delta \mid \theta(\alpha)=\alpha\} \\
\Delta_{r} & =\{\alpha \in \Delta \mid \theta(\alpha)=-\alpha\} \\
\Delta_{c x} & =\{\alpha \in \Delta \mid \theta(\alpha) \neq \pm \alpha\} .
\end{aligned}
$$


Then $\Delta_{i}, \Delta_{r}$ are root systems ( $\Delta_{c x}$ typically is not), and we denote their Weyl groups $W_{i}, W_{r}$.

Fix a set of positive roots $\Delta^{+}$. For $*=i, r$ or $c x$ let $\Delta_{*}^{+}=\Delta^{+} \cap \Delta_{*}$. Then $\Delta_{i}^{+}, \Delta_{r}^{+}$are positive roots for $\Delta_{i}, \Delta_{r}$, respectively. Define $\rho_{*}=\frac{1}{2} \sum_{\Delta_{*}^{+}} \alpha$.

We need to allow the positive systems for $\Delta_{i}, \Delta_{r}$ to vary independently of $\Delta^{+}$. We will write $\Psi_{i} \subset \Delta_{i}, \Psi_{r} \subset \Delta_{r}$ for choices of positive imaginary and real roots respectively, with $\rho_{i}, \rho_{r}$ defined accordingly. The covers $H(\mathbb{R})_{\rho_{i}}$ and $H(\mathbb{R})_{\rho_{r}}$ are defined as above.

Definition 2.4 If $\Delta^{+}$is a set of positive roots define

$$
\begin{aligned}
D^{0}\left(\Delta^{+}, g\right) & =\prod_{\alpha \in \Delta^{+}}\left(1-e^{-\alpha}(g)\right) \quad(g \in H), \\
D\left(\Delta^{+}, \widetilde{g}\right) & =D^{0}\left(\Delta^{+}, g\right) e^{\rho}(\widetilde{g}) \quad(\widetilde{g} \in \widetilde{H})
\end{aligned}
$$

where $g$ is the image of $\widetilde{g}$ in $H$, and

$$
\left|D\left(\Delta^{+}, g\right)\right|=\left|D^{0}\left(\Delta^{+}, g\right) e^{\rho}(\widetilde{g})\right| \quad(g \in H)
$$

where $\widetilde{g}$ is any inverse image of $g$ in $\widetilde{H}$.

The dependence of $D$ on $\Delta^{+}$is obvious (modulo chasing the covers a bit):

$$
D\left(\Delta^{+}, w^{-1} \widetilde{g}\right)=D\left(w \Delta^{+}, \widetilde{g}\right)=\operatorname{sgn}(w) D\left(\Delta^{+}, \widetilde{g}\right) \quad(w \in W) .
$$

It is also easy to see

$$
D\left(\Delta^{+}, \widetilde{g}\right)=\sum_{w \in W} \operatorname{sgn}(w)\left(w \times e^{\rho}\right)(\widetilde{g}) \quad\left(\widetilde{g} \in H_{\rho}\right) .
$$

Suppose $H$ is defined over $\mathbb{R}$. After conjugating by $G(\mathbb{R})$ we may assume $H$ is $\theta$-stable, which we often do without further comment. The real Weyl group is $W(G(\mathbb{R}), H(\mathbb{R}))=\operatorname{Norm}_{G(\mathbb{R})}(H(\mathbb{R})) / H(\mathbb{R})$. This is a subgroup of $W^{\theta}$, the elements of $W$ commuting with the action of $\theta$ on $H$.

Fix positive imaginary and real roots $\Psi_{i}, \Psi_{r}$. For $w \in W^{\theta}$ define $w_{i} \in$ $W\left(\Delta_{i}\right), w_{r} \in W\left(\Delta_{r}\right)$ by $w \Psi_{i}=w_{i} \Psi_{i}$ and $w \Psi_{r}=w_{r} \Psi_{r}$. Define $w_{c x}$ by $w=w_{c x} w_{i} w_{r}$. Then $w_{c x} \in W^{\theta}, w_{c x}$ fixes $\Psi_{i}, \Psi_{r}$, and $\operatorname{sgn}\left(w_{c x}\right)=1$. See $[22$, Section 3]. Define $\operatorname{sgn}_{i}(w)=\operatorname{sgn}\left(w_{i}\right)$. Although $w_{i}$ depends on the choice of $\Psi_{i}, \operatorname{sgn}\left(w_{i}\right)$ is independent of this choice. 
If $H$ is $\theta$-stable then $H=T A$ where $T=H^{\theta}$ and $A$ is the identity component of $\left\{h \in H \mid \theta(h)=h^{-1}\right\}$. Let $M=\operatorname{Cent}_{G}(A)$; this is a connected complex reductive group. If $H$ is defined over $\mathbb{R}$ then so are $T, A$ and $M$, and $M^{\theta}$ is a complexified maximal compact subgroup of $M(\mathbb{R})$. With $d$ denoting the derived group define

$$
\begin{aligned}
q_{H} & =\frac{1}{2} \operatorname{dim}_{\mathbb{C}}\left(M_{d} /\left(M_{d}\right)^{\theta}\right) \\
& =\frac{1}{2} \mid\left\{\alpha \in \Delta_{i} \mid \alpha \text { is noncompact }\right\} \mid .
\end{aligned}
$$

Fix a set of positive real roots $\Psi_{r}$. For later use we define functions $\epsilon\left(\Psi_{r}, g\right)= \pm 1, \gamma\left(\Psi_{r}\right)(\widetilde{g})= \pm 1, \pm i$ and $\tau\left(\Psi_{r}, w\right)(g)= \pm 1$, and give some elementary properties.

For $g \in H(\mathbb{R})$ define

$$
\epsilon\left(\Psi_{r}, g\right)=\operatorname{sgn} \prod_{\alpha \in \Psi_{r}}\left(1-e^{-\alpha}(g)\right) .
$$

View $e^{\rho_{r}}$ as a genuine character of $H(\mathbb{R})_{\rho_{r}}$ and define

$$
\gamma\left(\Psi_{r}\right)(\widetilde{g})=\frac{e^{\rho_{r}}(\widetilde{g})}{\left|e^{\rho_{r}}(\widetilde{g})\right|} \quad\left(\widetilde{g} \in H(\mathbb{R})_{\rho_{r}}\right) .
$$

Since $\left|e^{\rho_{r}}(\widetilde{g})\right|$ factors to $H(\mathbb{R})$, this is a genuine character of $H(\mathbb{R})_{\rho_{r}}$, and since $e^{2 \rho_{r}}(g) \in \mathbb{R}^{\times}, \gamma\left(\Psi_{r}\right)(\widetilde{g})^{4}=1$. For $w \in W^{\theta}$ define

$$
\tau\left(\Psi_{r}, w\right)(g)=\frac{\gamma\left(\Psi_{r}\right)}{\gamma\left(w \Psi_{r}\right)}(g)=\operatorname{sgn}\left(e^{\rho_{r}-w \rho_{r}}(g)\right) \quad(g \in H(\mathbb{R})) .
$$

As indicated this factors to a character on $H(\mathbb{R})$, with values in \pm 1 .

Lemma 2.12 For all $w, x, y \in W^{\theta}$ :

(1) $\tau\left(\Psi_{r}, w\right)=\tau\left(\Psi_{r}, w_{r}\right)$.

(2) $\tau\left(\Psi_{r}, x y\right)=\tau\left(\Psi_{r}, x\right) x \tau\left(\Psi_{r}, y\right)$;

(3) $\epsilon\left(\Psi_{r}, w g\right)=\operatorname{sgn}\left(w_{r}\right) \epsilon\left(\Psi_{r}, g\right) \tau\left(\Psi_{r}, w_{r}^{-1}\right)(g)$.

We omit the straightforward proofs. For $(2)$ see $[4,(8.26)(\mathrm{b})])$. 
Definition 2.13 Suppose $H$ is $\theta$-stable and $\Delta^{+}$is a set of positive roots. Let

$$
B\left(\Delta^{+}\right)=\frac{1}{2} \mid\left\{\alpha \in \Delta^{+} \text {complex } \mid \theta \alpha \in \Delta^{+}\right\} \mid .
$$

We make repeated use of the condition

$$
\alpha \in \Delta^{+} \text {complex } \Rightarrow \theta(\alpha) \notin \Delta^{+},
$$

i.e. $B\left(\Delta^{+}\right)=0$. See $[21$, Lemma 6.7.1].

Let $\bar{\alpha}$ denote the complex conjugate of $\alpha$. Then $\theta \alpha=-\bar{\alpha}$, so (2.15) holds if and only if the non-imaginary roots roots are closed under $\alpha \rightarrow \bar{\alpha}$, i.e. these are the roots of $\mathfrak{h}$ in $\mathfrak{n}$, where $M N$ is a parabolic subgroup defined over $\mathbb{R}$.

Suppose $\alpha$ is a complex root. Then $e^{\alpha+\bar{\alpha}}$ takes positive real values on $H(\mathbb{R})$, and has a natural positive square-root. Accordingly for $h \in H(\mathbb{R})$ we define

$$
\begin{aligned}
e^{\frac{1}{2}(\alpha+\bar{\alpha})}(h) & =\sqrt{e^{\alpha+\bar{\alpha}}(h)} \\
e^{\frac{1}{2}(\alpha-\bar{\alpha})}(h) & =e^{\alpha}(h) e^{-\frac{1}{2}(\alpha+\bar{\alpha})}(h) .
\end{aligned}
$$

Since $\rho_{c x}$ is a sum of terms $\frac{1}{2}(\alpha \pm \bar{\alpha}), e^{\rho_{c x}}$ is a well defined character of $H(\mathbb{R})$. In particular if $\Delta^{+}$satisfies $(2.15)$ then $e^{\rho_{c x}}(h)>0$ for $h \in H(\mathbb{R})$.

Suppose $\Lambda$ is a character of $\widetilde{H(\mathbb{R})}$, and $\left\langle d \Lambda, \alpha^{\vee}\right\rangle \in \mathbb{R}_{\neq 0}$ for all roots. We define

$$
\epsilon\left(\Lambda, \Delta^{+}\right)=\operatorname{sgn}(w) \quad \text { where }\left\langle w d \Lambda, \alpha^{\vee}\right\rangle>0 \text { for all } \alpha \in \Delta^{+} .
$$

We often apply this to $\Delta_{i}^{+}$in which case it is enough to assume $\left\langle d \Lambda, \alpha^{\vee}\right\rangle \neq 0$ for all imaginary roots.

\section{$3 \quad$ Limits of Relative Discrete Series}

We say a Cartan subgroup $H$ (defined over $\mathbb{R}$ ) is relatively compact if $H(\mathbb{R})$ is compact modulo the center of $G(\mathbb{R})$. By a result of Harish-Chandra $G(\mathbb{R})$ has relative discrete series representations (i.e. discrete series modulo the center) if and only if it has a relatively compact Cartan subgroup. It also has limits of such representations, which are obtained by translating to singular infinitesimal character.

$$
\text { Let } q=q_{H}=\frac{1}{2} \operatorname{dim}_{\mathbb{C}}\left(G_{d} /\left(G_{d}\right)^{\theta}\right)(\text { cf. 2.8). }
$$


Definition 3.1 A relative discrete series parameter is a pair $\gamma=(H, \Lambda)$ where $H$ is relatively compact, $\Lambda$ is a genuine character of $H(\mathbb{R})$ and

$$
\left\langle d \Lambda, \alpha^{\vee}\right\rangle \neq 0 \quad \text { for all } \alpha \in \Delta(G, H) .
$$

Let $\Delta^{+}=\Delta^{+}(d \Lambda)=\left\{\alpha \mid\left\langle d \Lambda, \alpha^{\vee}\right\rangle>0\right\}$.

Let $I(\gamma)$ be the unique relative discrete series representation whose character restricted to the regular elements of $H(\mathbb{R})$ is

$$
\Theta_{I(\gamma)}(g)=(-1)^{q} D\left(\Delta^{+}, \widetilde{g}\right)^{-1} \sum_{w \in W(G(\mathbb{R}), H(\mathbb{R}))} \operatorname{sgn}(w)(w \Lambda)(\widetilde{g})
$$

where $\widetilde{g} \in \widetilde{H(\mathbb{R})}$ maps to $g$.

More generally let $\gamma=\left(H, \Delta^{+}, \Lambda\right)$ where $\Lambda$ is a genuine character of $H(\mathbb{R})$ satisfying

$$
\left\langle d \Lambda, \alpha^{\vee}\right\rangle \neq 0 \quad \text { if } \alpha \text { is compact and simple for } \Delta^{+},
$$

and $\Delta^{+}$satisfies

$$
\left\{\alpha \mid\left\langle d \Lambda, \alpha^{\vee}\right\rangle>0\right\} \subset \Delta^{+} .
$$

Choose $\Lambda^{\prime}$ regular so that $\Lambda^{\prime}-\Lambda$ is a sum of roots, and satisfying $\Delta^{+}=$ $\Delta^{+}\left(\Lambda^{\prime}\right)$. Then $I\left(H, \Lambda^{\prime}\right)$ is a discrete series representation by the preceding construction. Define $I\left(H, \Delta^{+}, \Lambda\right)=\Psi\left(I\left(H, \Lambda^{\prime}\right)\right)$ where $\Psi$ is the JantzenZuckerman translation functor taking infinitesimal character $d \Lambda^{\prime}$ to infinites-

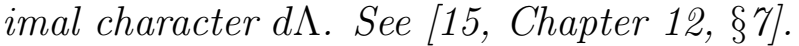

Every relative limit of discrete series representation is obtained this way, and $I(\gamma) \simeq I\left(\gamma^{\prime}\right)$ if and only if $\gamma$ is $G(\mathbb{R})$ conjugate to $\gamma^{\prime}$.

See [4, Example 8.14].

Formula (3.3) holds for limits of discrete series. However the uniqueness statement does not. For example for $S O(2,1)$ the character of the (unique) limit of discrete series representation vanishes on the compact Cartan subgroup.

\section{Regular Integral Standard Modules}

In this section we consider standard modules with regular integral infinitesimal character. The basic reference for this section and the next is $[4$, Section 8]. Also see [3, Theorem 11.4] and [1, Theorem 6.1]. 
We first dispense with a technical issue about the covers. Suppose $H$ is $\theta$-stable, $\Delta^{+}$is a set of positive roots, and define $\rho_{i}, \rho_{r}, \rho_{c x}$ with respect to $\Delta_{i}^{+}, \Delta_{r}^{+}$and $\Delta_{c x}^{+}$as in Section 2. Recall (end of Section 2) $e^{\rho_{c x}}$ is a well defined character of $H(\mathbb{R})$.

Definition 4.1 Fix $H$ (defined over $\mathbb{R}$ ) and $\Delta^{+}$. Suppose $\chi, \tau$ are genuine characters of $H(\mathbb{R})_{\rho}, H(\mathbb{R})_{\rho_{r}}$, respectively. Define:

$$
(\chi \otimes \tau)(\bar{h})=\left(\chi e^{-\rho}\right)(h)\left(\tau e^{\rho_{r}}\right)(h) e^{\rho_{c x}}(h) e^{\rho_{i}}(\bar{h}) \quad\left(\bar{h} \in H(\mathbb{R})_{\rho_{i}}\right)
$$

where $h$ is the image of $\bar{h}$ in $H(\mathbb{R})$. This is a genuine character of $H(\mathbb{R})_{\rho_{i}}$, satisfying $d(\chi \otimes \tau)=d \chi+d \tau$.

Now suppose $\Lambda$ is a genuine character of $\widetilde{H(\mathbb{R})}$. Fix positive imaginary and real roots $\Psi_{i}, \Psi_{r}$. Choose positive roots $\Delta^{+}$containing $\Psi_{i}, \Psi_{r}$, and apply Definition 4.1 to define (cf. (2.10))

$$
\Lambda_{M}\left(\Psi_{r}\right)=\Lambda \otimes \gamma\left(\Psi_{r}\right)^{-1},
$$

a genuine character of $H(\mathbb{R})_{\rho_{i}}$. It is easy to see this is independent of the choice of $\Delta^{+}$, and the dependence on $\Psi_{r}$ is given by

$$
\Lambda_{M}\left(w \Psi_{r}\right)=\tau\left(\Psi_{r}, w\right) \Lambda_{M}\left(\Psi_{r}\right) \quad\left(w \in W_{r}\right) .
$$

Definition 4.5 A regular character for $G(\mathbb{R})$ is a pair $\gamma=(H, \Lambda)$ where $H$ is a Cartan subgroup of $G$, defined over $\mathbb{R}, \Lambda$ is a genuine character of $\widetilde{H(\mathbb{R})}$, and $d \Lambda$ is regular and integral, i.e. $\left\langle d \Lambda, \alpha^{\vee}\right\rangle \in \mathbb{Z}_{\neq 0}$ for all roots.

We say $\gamma=(H, \Lambda)$ is based on $H$. The group $G(\mathbb{R})$ acts on regular characters by conjugation.

Given $\gamma=(H, \Lambda)$, conjugate by $G(\mathbb{R})$ as usual to assume $H(\mathbb{R})$ is $\theta$ stable. Write $H=T A$ and $M=\operatorname{Cent}_{G}(A)$ as in Section 2. Let $\mathfrak{a}=\operatorname{Lie}(A)$. Recall $M$ is defined over $\mathbb{R}$. We want to define a real parabolic subgroup $P(\mathbb{R})=M(\mathbb{R}) N(\mathbb{R})$. Let

$$
\Psi_{r}=\left\{\alpha \in \Delta_{r} \mid\left\langle d \Lambda, \alpha^{\vee}\right\rangle>0\right\} .
$$

Let $Q=L U$ be the parabolic subgroup such that the weights of $\mathfrak{h}$ in the Lie algebra of $P$ are

$$
\left\{\alpha \mid\left\langle\left. d \Lambda\right|_{\mathfrak{a}}, \alpha^{\vee}\right\rangle \geq 0\right\}
$$


This is defined over $\mathbb{R}$. However $L$ may be larger than $M$. Choose a parabolic subgroup $P_{L}=M N_{L}$ of $M$, defined over $\mathbb{R}$ and let $N=N_{L} U$. Then $P(\mathbb{R})=$ $M(\mathbb{R}) N(\mathbb{R})$ is is a parabolic subgroup of $G(\mathbb{R})$.

Definition 4.8 Suppose $\gamma=(H, \Lambda)$ is a regular character. Define $\Psi_{r}$ by (4.6), $\Lambda_{M}$ by by (4.3), and the relative discrete series representation $I_{M}\left(H, \Lambda_{M}\right)$ by Definition 3.1 applied to $M(\mathbb{R})$. Choose $P(\mathbb{R})$ as above, and define

$$
I(\gamma)=\operatorname{Ind} d_{P(\mathbb{R})}^{G(\mathbb{R})}\left(I_{M}\left(H, \Lambda_{M}\right)\right) .
$$

By induction in stages this is independent of the choice of $P(\mathbb{R})$, due to the fact that the induction from $P_{L}(\mathbb{R})$ to $L(\mathbb{R})$ is unitary (modulo the center), and hence independent of the choice of $N_{L}$.

Theorem 4.10 I $(\gamma)$ is non-zero and has a unique irreducible quotient, denoted $\pi(\gamma)$. Every irreducible representation of $G(\mathbb{R})$ with regular integral infinitesimal character is isomorphic to $\pi(\gamma)$ for some regular character $\gamma$, unique up to conjugacy by $G(\mathbb{R})$.

Example 4.11 Let $G(\mathbb{R})=S L(2, \mathbb{R})$ and let $H(\mathbb{R})$ be a split Cartan subgroup. Choose an isomorphism $h: \mathbb{R}^{*} \simeq H(\mathbb{R})$. The cover $H(\mathbb{R})_{\rho}$ is trivial, so we may drop it from the notation. Let $\Psi_{r}=\{\alpha\}$ where $\alpha(h(x))=\operatorname{sgn}(x)$. Then $e^{\rho_{r}}(h(x))=x$ and $\gamma\left(\Psi_{r}\right)(h(x))=\operatorname{sgn}(x)$. If $\Lambda$ is a character of $H(\mathbb{R})$ then $\Lambda_{M}(h(x))=\Lambda(h(x)) \operatorname{sgn}(x)$. In particular if $\Lambda=e^{\rho}$ then $\Lambda_{M}(h(x))=$ $|x|$.

Example 4.12 Consider the split Cartan subgroup $H(\mathbb{R}) \simeq \mathbb{R}^{*}$ of $P G L(2, \mathbb{R})$. Now some care is required with the covers. Choose an isomorphism $h: \mathbb{R}^{*} \simeq$ $H(\mathbb{R})$, and choose $\Psi_{r}=\{\alpha\}$ where $\alpha(h(x))=x$. Then $H(\mathbb{R})_{\rho}=H(\mathbb{R})_{\rho_{r}}=$ $\left\{(h(x), z) \mid z^{2}=x\right\}$. On the other hand $H(\mathbb{R})_{\rho_{i}}=\left\{(h(x), \epsilon) \mid \epsilon^{2}=1\right\}$ (trivial cover).

Suppose $\Lambda$ is a genuine character of $H(\mathbb{R})_{\rho}$. Write $\Lambda(h(x), z)=\mu(x) z$ for $\mu$ a character of $\mathbb{R}^{*}$. Note that $\gamma\left(\Psi_{r}\right)(h, z)=z /|z|$. According to Definition 4.1 if $(h, \epsilon) \in H(\mathbb{R})_{\rho_{i}}$ :

$$
\begin{aligned}
\Lambda_{M}(h(x), \epsilon) & =\left(\Lambda e^{-\rho}\right)(h(x))\left(\gamma\left(\Psi_{r}\right)^{-1} e^{\rho_{r}}\right)(h(x)) \epsilon \\
& =\mu(x)|z| \epsilon=\mu(x)|x|^{\frac{1}{2}} \epsilon .
\end{aligned}
$$

We may ignore the trivial cover $H(\mathbb{R})_{\rho_{i}}$ and write $\Lambda_{M}(h(x))=\mu(x)|x|^{\frac{1}{2}}$. In particular $\mu=1$ gives $\Lambda=e^{\rho}$ and $\Lambda_{M}(h(x))=|x|^{\frac{1}{2}}$. 


\section{$5 \quad$ General Standard Modules}

A general standard module is obtained by dropping the assumption that $d \Lambda$ is regular and integral, in which case some further choices are needed. First of all we need to choose $\Psi_{i}$ to define limits of discrete series for $M(\mathbb{R})$. Secondly $\Psi_{r}$ is not determined by (4.6), so we need to choose $\Psi_{r}$ to define $\Lambda_{M}$. As in the previous section see [4, Section 8], [3, Theorem 11.4] and [1, Theorem 6.1] for details.

Definition 5.1 Standard limit data is a set $\gamma=\left(H, \Psi_{i}, \Psi_{r}, \Lambda\right)$ where $H$ is defined over $\mathbb{R}, \Psi_{i}, \Psi_{r}$ are sets of positive imaginary and real roots, respectively, and $\Lambda$ is a genuine character of $\widehat{H(\mathbb{R})}$. We require:

$$
\left\{\alpha \in \Delta_{i} \mid\left\langle d \Lambda, \alpha^{\vee}\right\rangle>0\right\} \subset \Psi_{i}
$$

if $\alpha \in \Delta_{i}$ is compact then $\left\langle d \Lambda, \alpha^{\vee}\right\rangle \neq 0$.

We say $\gamma=\left(H, \Psi_{i}, \Psi_{r}, \Lambda\right)$ is based on $H$. The group $G(\mathbb{R})$ acts on standard limit data by conjugation.

Definition 5.3 Suppose $\gamma=\left(H, \Psi_{i}, \Psi_{r}, \Lambda\right)$ is standard limit data. Define $\Lambda_{M}$ by (4.3) and $I_{M}\left(H, \Psi_{i}, \Lambda_{M}\right)$ by Definition 3.1 applied to $M$.

Let $P=M N$ be a real parabolic subgroup such that

$$
\operatorname{Re}\left\langle\left. d \Lambda\right|_{\mathfrak{a}}, \alpha^{\vee}\right\rangle \geq 0 \quad \text { for all roots of } \mathfrak{h} \text { in } \operatorname{Lie}(P) \text {. }
$$

Define

$$
I(\gamma)=\operatorname{Ind} d_{P(\mathbb{R})}^{G(\mathbb{R})}\left(I_{M}\left(H, \Psi_{i}, \Lambda_{M}\left(\Psi_{r}\right)\right)\right) .
$$

If a complex root $\alpha$ satisfies (5.4) then so does $\bar{\alpha}=-\theta \alpha$. It follows that we can choose $N$ so that it, and hence $P$, are defined over $\mathbb{R}$.

By the induced character formula the character of $I(\gamma)$ is independent of the choice of $N(\mathbb{R})$ regardless of condition (5.4). Furthermore it is a basic fact that the module itself is independent of $N(\mathbb{R})$ provided (5.4) holds. See [4, Section 8] for details.

If $d \Lambda$ is regular and integral we recover Definition 4.5:

$$
I(H, \Lambda)=I\left(H, \Delta_{i}^{+}, \Delta_{r}^{+}, \Lambda\right)
$$


where $\Delta^{+}=\left\{\alpha \mid\left\langle d \Lambda, \alpha^{\vee}\right\rangle>0\right\}$. More generally suppose $\left\langle d \Lambda, \alpha^{\vee}\right\rangle \neq 0$ for all imaginary roots. Then $\Psi_{i}$ is determined by (5.2)(a), and we define

$$
I\left(H, \Psi_{r}, \Lambda\right)=I\left(H, \Psi_{i}, \Psi_{r}, \Lambda\right)
$$

where $\Psi_{i}=\left\{\alpha \in \Delta_{i}, \mid\left\langle d \Lambda, \alpha^{\vee}\right\rangle>0\right\}$.

Definition 5.8 Fix $\gamma=\left(H, \Psi_{i}, \Psi_{r}, \Lambda\right)$. We say standard limit data $\gamma^{\prime}$ is equivalent to $\gamma$ if there exists $g \in G(\mathbb{R}), w \in W_{r}$ such that

$$
g \gamma^{\prime} g^{-1}=\left(H, \Psi_{i}, w \Psi_{r}, \tau\left(\Psi_{r}, w\right) \Lambda\right) .
$$

In particular $\left(H, \Psi_{i}, \Psi_{r}, \Lambda\right) \sim\left(H, \Psi_{i}^{\prime}, \Psi_{r}^{\prime}, \Lambda^{\prime}\right)$ if and only if there exists $w \in$ $W(G(\mathbb{R}), H(\mathbb{R}))$ such that

(1) $\Psi_{i}=w \Psi_{i}^{\prime}$

(2) $\Lambda=\tau\left(\Psi_{r}, y\right) w \Lambda^{\prime}$ where $y \in W_{r}$ satisfies $y \Psi_{r}=w \Psi_{r}^{\prime}$.

Theorem 5.10 Suppose $\gamma, \gamma^{\prime}$ are standard limit data. Then $I(\gamma) \simeq I\left(\gamma^{\prime}\right)$ if $\gamma \sim \gamma^{\prime}$.

Every irreducible representation is a quotient of some $I(\gamma)$. If the infinitesimal character is regular then $\gamma$ is determined uniquely up to equivalence. For singular infinitesimal character a further final condition on standard limit data is required to avoid overlaps. We will not need this condition. See [3, Definition 11.13, Condition (b)] or [1, Theorem 6.1, Condition 5].

A virtual character is an element of the Grothendieck group, i.e. a finite linear combination of irreducible representations, with integral coefficients. A basic result is that the representations $I(\gamma)$ span the virtual characters. At regular infinitesimal character these form a basis (at singular infinitesimal character this is not true without the final condition of the previous paragraph). We restrict to regular infinitesimal character.

Definition 5.11 For $\pi$ a virtual representation with regular infinitesimal character define integers $M(I(\gamma), \pi)$ by

$$
\pi=\sum_{\gamma} M(I(\gamma), \pi) I(\gamma)
$$

in the Grothendieck group.

For $\pi$ irreducible the integers $M(I(\gamma), \pi)$ are computed by the KazhdanLusztig-Vogan algorithm. 


\section{Character Formulas}

Fix a Cartan subgroup $H$ defined over $\mathbb{R}$ and write $H(\mathbb{R})_{\text {reg }}$ for the regular elements of $H(\mathbb{R})$. Fix a set of positive real roots $\Psi_{r}$. Let

$$
H(\mathbb{R})_{+}=\left\{g \in H(\mathbb{R})_{r e g}|| e^{\alpha}(g) \mid>1 \text { for all } \alpha \in \Psi_{r}\right\} .
$$

Every element of $H(\mathbb{R})_{\text {reg }}$ is conjugate via $W_{r} \subset W(G(\mathbb{R}), H(\mathbb{R}))$ to a unique element of $H(\mathbb{R})_{+}$.

We parametrize infinitesimal characters by Weyl group orbits of elements of $\mathfrak{h}^{*}$. Let

$$
\mathcal{P}(H, \lambda)=\{\Lambda \mid \Lambda \text { is a genuine character of } \widetilde{H(\mathbb{R})}, d \Lambda \in W \lambda\} .
$$

Since we make frequent use of the trivial representation, let $\rho$ be one-half the sum of any set of positive roots and define

$$
\mathcal{P}(H, \mathbb{C})=\mathcal{P}(H, \rho)
$$

Proposition 6.4 Suppose $\pi$ is an admissible representation. Fix a Cartan subgroup $H$, defined over $\mathbb{R}$, and a set of positive roots $\Delta^{+}$. Suppose $\pi$ has infinitesimal character $\lambda \in \mathfrak{h}^{*}$. Use $\Delta_{r}^{+}$to define $H(\mathbb{R})_{+}$. Then the restriction of $\Theta_{\pi}$ to $H(\mathbb{R})_{+}$may be written

$$
\theta_{\pi}(g)=\frac{\sum_{\Lambda \in \mathcal{P}(H, \lambda)} a\left(\pi, \Delta^{+}, \Lambda\right) \Lambda(\widetilde{g})}{D\left(\Delta^{+}, \widetilde{g}\right)}
$$

for some unique integers $a\left(\pi, \Delta^{+}, \Lambda\right)$.

Here $\widetilde{g}$ is an inverse image of $g$ in $\widetilde{H(\mathbb{R})}$; the right hand side is independent of this choice.

This is essentially due to Harish-Chandra [7], see [10, 3.41], except that the set on which this expansion is valid is larger. Also see see [1, Theorem 5.8] and [20].

We want to compute the integers $a\left(\pi, \Delta^{+}, \Lambda\right)$.

From invariance of $\theta_{\pi}$ it it is easy to see that if $w \in W(G(\mathbb{R}), H(\mathbb{R}))$ then

$$
a\left(\pi, w \Delta^{+}, w \Lambda\right)=a\left(\pi, \Delta^{+}, \Lambda\right)
$$


and if furthermore $w \Delta_{r}^{+}=\Delta_{r}^{+}$, then (cf. (2.6))

$$
a\left(\pi, \Delta^{+}, w \Lambda\right)=\operatorname{sgn}(w) a\left(\pi, \Delta^{+}, \Lambda\right) .
$$

However if $w \in W_{r}$ there is often no relationship between $a\left(\pi, \Delta^{+}, \Lambda\right)$ and $a\left(\pi, \Delta^{+}, w \Lambda\right)$.

The dependence of $a\left(\pi, \Delta^{+}, \Lambda\right)$ on $\Delta^{+}$is fairly innocuous, and we record it here. Recall if $\Delta^{+}$is any set of positive roots then $\Delta_{r}^{+}=\Delta^{+} \cap \Delta_{r}$ is a set of positive roots of $\Delta_{r}$.

Lemma 6.7 Fix a $\theta$-stable Cartan subgroup $H, \Delta^{+}$, and suppose $w \in W$. There exist unique $x, y \in W$ such that

$$
\begin{aligned}
x \in W_{r} \text { and }\left(w \Delta^{+}\right)_{r} & =x\left(\Delta_{r}^{+}\right), \\
\left(y \Delta^{+}\right)_{r} & =\Delta_{r}^{+}, \\
w & =x y .
\end{aligned}
$$

If $w \in W^{\theta}$ then $\left(w \Delta^{+}\right)_{r}=w\left(\Delta_{r}^{+}\right)$and $x=w_{r}$ (cf. Section 2).

Proof. The element $x$ satisfying (a) exists and is unique, so let $y=x^{-1} w$. It is enough to show $y$ satisfies (b). Note that $\left(x^{-1} w \Delta^{+}\right)_{r}=x^{-1}\left[\left(w \Delta^{+}\right)_{r}\right]$ since $x \in W_{r}$. Thus

$$
\left(y \Delta^{+}\right)_{r}=\left(x^{-1} w \Delta^{+}\right)_{r}=x^{-1}\left[\left(w \Delta^{+}\right)_{r}\right]=x^{-1}\left[x\left(\Delta_{r}^{+}\right)\right]=\Delta_{r}^{+} .
$$

Lemma 6.10 In the setting of Proposition 6.4 suppose $w \in W$, and write $w=x y$ as in the Lemma. Then

$$
a\left(\pi, w \Delta^{+}, \Lambda\right)=\operatorname{sgn}(y) a\left(\pi, \Delta^{+}, x^{-1} \Lambda\right) .
$$

In particular

$$
\begin{aligned}
& a\left(\pi, w \Delta^{+}, \Lambda\right)=\operatorname{sgn}(w) a\left(\pi, \Delta^{+}, \Lambda\right) \quad\left(w \Delta^{+}\right)_{r}=w\left(\Delta_{r}^{+}\right) \\
& a\left(\pi, w \Delta^{+}, \Lambda\right)=a\left(\pi, \Delta^{+}, w^{-1} \Lambda\right) \quad\left(w \in W_{r}\right) .
\end{aligned}
$$

In particular (b) holds for $w \in W_{i}$. 
Proof. Assuming $\left|e^{\alpha}(g)\right|>1$ for all $\alpha \in \Delta_{r}^{+}$, we have

$$
\Theta_{\pi}(g)=D\left(\Delta^{+}, \widetilde{g}\right)^{-1} \sum a\left(\pi, \Delta^{+}, \Lambda\right) \Lambda(\widetilde{g}) .
$$

Since $\left(w \Delta^{+}\right)_{r}=x\left(\Delta_{r}^{+}\right), g$ satisfies the previous condition if and only if $\left|e^{\beta}(x g)\right|>1$ for all $\beta \in\left(w \Delta^{+}\right)_{r}$. Therefore

$$
\begin{aligned}
\Theta_{\pi}(x g) & =D\left(w \Delta^{+}, x \widetilde{g}\right)^{-1} \sum a\left(\pi, w \Delta^{+}, \Gamma\right) \Gamma(x \widetilde{g}) \\
& =\operatorname{sgn}(w) \operatorname{sgn}(x) D\left(\Delta^{+}, \widetilde{g}\right)^{-1} \sum a\left(\pi, w \Delta^{+}, \Gamma\right)\left(x^{-1} \Gamma\right)(\widetilde{g}) \\
& =\operatorname{sgn}(y) D\left(\Delta^{+}, \widetilde{g}\right)^{-1} \sum a\left(\pi, w \Delta^{+}, x \Lambda\right) \Lambda(\widetilde{g})
\end{aligned}
$$

Setting $\theta_{\pi}(g)=\theta_{\pi}(x g)$ gives the result.

It makes sense to use $(6.11)$ (b) to move $\Lambda$ to the dominant $\Delta_{i}^{+}$-chamber using $W_{i}$, which (provided $d \Lambda$ is regular) introduces a term $\epsilon\left(\Lambda, \Delta_{i}^{+}\right.$) (see the end of Section 2).

It is also useful to record how $a\left(\pi, \Delta^{+}, \Lambda\right)$ depends on $\Delta^{+}$provided $\Delta_{i}^{+}, \Delta_{r}^{+}$are fixed. Recall (Definition 2.13) $B\left(\Delta^{+}\right)=\frac{1}{2} \mid\left\{\alpha \in \Delta^{+}\right.$complex $\mid \theta \alpha \in$ $\left.\Delta^{+}\right\} \mid$.

Lemma 6.14 Assume $\Delta_{1}^{+}, \Delta_{2}^{+}$are sets of positive roots, containing the same real and imaginary roots. Then

$$
a\left(\pi, \Delta_{1}^{+}, \Lambda\right)=(-1)^{B\left(\Delta_{1}^{+}\right)-B\left(\Delta_{2}^{+}\right)} a\left(\pi, \Delta_{2}^{+}, \Lambda\right) .
$$

Proof. Choose $w$ such that $w \Delta_{1}^{+}=\Delta_{2}^{+}$. By Lemma $6.10 a\left(\pi, \Delta_{1}^{+}, \Lambda\right)=$ $(-1)^{\ell(w)} a\left(\pi, \Delta_{2}^{+}, \Lambda\right)$. It is easy to see $\ell(w)=B\left(\Delta_{1}^{+}\right)-B\left(\Delta_{2}^{+}\right)(\bmod 2)$.

Example 6.16 Suppose $H$ is a relatively compact Cartan subgroup, and $I(H, \Lambda)$ is a relative discrete series representation (Section 3). Then with $q_{H}$ as in (2.8),

$$
a\left(I(H, \Lambda), \Delta^{+}, \Gamma\right)= \begin{cases}(-1)^{q_{H}} \epsilon\left(\Gamma, \Delta^{+}\right) & \Gamma \text { is } G(\mathbb{R})-\text { conjugate to } \Lambda \\ 0 & \text { otherwise. }\end{cases}
$$

For a more familiar version assume $d \Lambda$ is $\Delta^{+}$-dominant. Then

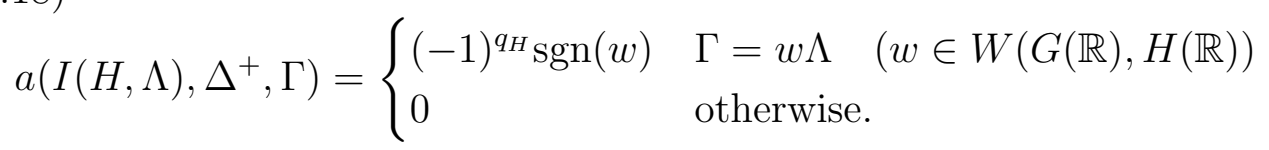




\section{Example: The Trivial Representation}

The character of the trivial representation $\mathbb{C}$ is the identity function. By (2.7):

$$
\theta_{\mathbb{C}}(g)=D\left(\Delta^{+}, \widetilde{g}\right)^{-1} \sum_{w \in W} \operatorname{sgn}(w)\left(w \times e^{\rho}\right)(\widetilde{g}) \quad\left(\widetilde{g} \in H(\mathbb{R})_{\rho}\right) .
$$

We reinterpret this formula in terms of holomorphic characters. The group $H_{\rho}$ inherits from $H$ a holomorphic structure. Therefore it makes sense to talk about holomorphic characters of $H_{\rho}$. The character $\rho$ is holomorphic, so the genuine holomorphic characters of $H_{\rho}$ are precisely the holomorphic characters of $H$, (pulled back to $H_{\rho}$ and) tensored with $\rho$.

It is clear from the definition of the cross action that if $\Lambda$ is holomorphic then $w \times \Lambda$ is (defined and) holomorphic for all $w \in W$. It is also clear that for $w \in W, w \times e^{\rho}$ is the unique genuine holomorphic character of $H(\mathbb{R})_{\rho}$ with differential $w \rho$. Thus $\Lambda$ occurs in the character formula of the trivial representation $d \Lambda \in W \rho$ and $\Lambda$ is holomorphic. Recall (6.3) $\mathcal{P}(H, \mathbb{C})$ is the set of genuine characters of with differential in $W \rho$. So if we let

$$
\mathcal{P}^{\text {hol }}(H, \mathbb{C})=\{\Lambda \in \mathcal{P}(H, \mathbb{C}) \mid \Lambda \text { is holomorphic }\}
$$

we may reformulate $(7.1)$ as

$$
\theta_{\mathbb{C}}=D\left(\Delta^{+}\right)^{-1} \sum_{\Lambda \in \mathcal{P h o l}(H, \mathbb{C})} \epsilon\left(\Lambda, \Delta^{+}\right) \Lambda
$$

(cf. (2.17)). In other words:

Corollary 7.3 Fix $H$ and $\Delta^{+}$. Then

$$
a\left(\mathbb{C}, \Delta^{+}, \Lambda\right)= \begin{cases}\epsilon\left(\Lambda, \Delta^{+}\right) & \Lambda \in \mathcal{P}^{\text {hol }}(H, \mathbb{C}) \\ 0 & \text { otherwise }\end{cases}
$$

Equivalently

$$
a\left(\mathbb{C}, \Delta^{+}, w \times e^{\rho}\right)=\operatorname{sgn}(w)
$$

and all other $a\left(\mathbb{C}, \Delta^{+}, \Lambda\right)$ are 0 . 


\section{Character formula for standard modules}

Suppose $\gamma$ is standard limit data based on $H$. We give a formula for the character of $I(\gamma)$ on $H(\mathbb{R})$. This combines the character formula for (relative) discrete series on a (relatively) compact Cartan subgroup (3.3) with a special case of the induced character formula, which we now state. See [10, Theorem $5.7]$.

Proposition 8.1 Suppose $H$ is a Cartan subgroup, defined over $\mathbb{R}$, and $P=M N$ is a parabolic subgroup defined over $\mathbb{R}$ as usual. Suppose $\sigma$ is an admissible representation of $M(\mathbb{R})$, pulled back to $P(\mathbb{R})$. Let $\pi=\operatorname{Ind}_{P(\mathbb{R})}^{G(\mathbb{R})}(\sigma \otimes 1)$ (normalized induction). Then (for any choice of positive roots $\Delta^{+}$)

$$
\Theta_{\pi}(g)=\left|D\left(\Delta^{+}, g\right)\right|^{-1} \sum_{w}\left|D\left(\Delta_{i}^{+}, w g\right)\right| \Theta_{\sigma}(w g) \quad\left(g \in H(\mathbb{R})_{r e g}\right)
$$

where the sum is over $W(M(\mathbb{R}), H(\mathbb{R})) \backslash W(G(\mathbb{R}), H(\mathbb{R}))$.

Here is the main result of this section. Recall $q_{H}$ is given in $(2.8), B\left(\Delta^{+}\right)$ is given by (2.14), and (see Section 2) for $w \in W^{\theta} \operatorname{sgn}_{i}(w)$ is defined.

Proposition 8.3 Suppose $\gamma=\left(H, \Psi_{i}, \Psi_{r}, \Lambda\right)$ is standard limit data. Choose positive roots $\Delta^{+}$containing $\Psi_{i}$ and $\Psi_{r}$ and set $\delta=(-1)^{q_{H}+B\left(\Delta^{+}\right)}$. Then for $g \in H(\mathbb{R})_{\text {reg }}$

$$
\Theta_{I(\gamma)}(g)=\delta \frac{\epsilon\left(\Psi_{r}, g\right)}{D\left(\Delta^{+}, \widetilde{g}\right)} \sum_{w \in W(G(\mathbb{R}), H(\mathbb{R}))} \operatorname{sgn}_{i}(w) \tau\left(\Psi_{r}, w\right)(g)(w \Lambda)(\widetilde{g})
$$

where $\widetilde{g}$ is an inverse image of $g$ in $\widetilde{H(\mathbb{R})}$. In other words for $\Lambda$ a genuine character of $\widetilde{H(\mathbb{R})}$ :

$$
a\left(I(\gamma), \Delta^{+}, \Gamma\right)=\left\{\begin{array}{ll}
\delta \operatorname{sgn}_{i}(w) & \Gamma=\tau\left(\Psi_{r}, w\right)(w \Lambda) \\
0 & \text { otherwise }
\end{array} \quad(w \in W(G(\mathbb{R}), H(\mathbb{R}))\right.
$$

Remark 8.5 The fact that the right hand side of (8.4) is invariant under $g \rightarrow w g$ for $w \in W(G(\mathbb{R}), H(\mathbb{R})$ ) follows from (and is essentially equivalent to) Lemma 2.12 . 
Proof of Proposition 8.3. Write $W_{G}=W(G(\mathbb{R}), H(\mathbb{R}))$ and $W_{M}=$ $W(M(\mathbb{R}), H(\mathbb{R}))$. Recall $I\left(H, \Psi_{i}, \Psi_{r}, \Lambda\right)$ is induced from $I_{M}\left(H, \Psi_{i}, \Lambda_{M}\left(\Psi_{r}\right)\right)$ where $\Lambda_{M}\left(\Psi_{r}\right)$ is given by (4.3). To save a little space write $\Lambda_{M}=\Lambda_{M}\left(\Psi_{r}\right)$. By Proposition 8.3 and (3.3):

$$
\begin{aligned}
\Theta_{I(\gamma)}(g)= & \left|D\left(\Delta^{+}, g\right)\right|^{-1} \sum_{w \in W_{M} \backslash W_{G}}\left|D\left(\Psi_{i}, w g\right)\right| \Theta_{I_{M}\left(H, \Psi_{i}, \Lambda_{M}\right)}(w g) \\
= & \left|D\left(\Delta^{+}, g\right)\right|^{-1} \sum_{w \in W_{M} \backslash W_{G}}\left|D\left(\Psi_{i}, w g\right)\right| \times \\
& (-1)^{q_{H}} D\left(\Psi_{i}, \overline{w g}\right)^{-1} \sum_{y \in W_{M}} \operatorname{sgn}(y)\left(y \Lambda_{M}\right)(\overline{w g}) .
\end{aligned}
$$

Here $\overline{w g}$ is an inverse image of $w g$ in $H(\mathbb{R})_{\rho_{i}}$. Also choose an inverse image $\widetilde{g}$ of $g$ in $H(\mathbb{R})_{\rho}$. This gives

$$
(-1)^{q_{H}} D\left(\Delta^{+}, \widetilde{g}\right)^{-1} \sum_{y, w} \frac{D\left(\Delta^{+}, \widetilde{g}\right)}{\left|D\left(\Delta^{+}, g\right)\right|} \frac{\left|D\left(\Psi_{i}, w g\right)\right|}{D\left(\Psi_{i}, \overline{w g}\right)} \operatorname{sgn}(y) \Lambda_{M}\left(y^{-1} \overline{w g}\right) .
$$

Using $D\left(\Psi_{i}, y^{-1} \overline{w g}\right)=\operatorname{sgn}(y) D\left(\Psi_{i}, \overline{w g}\right)$ and $D\left(\Delta^{+}, \widetilde{g}\right)=\operatorname{sgn}(w) D\left(\Delta^{+}, w \widetilde{g}\right)$ we can combine the sums:

$$
(-1)^{q_{H}} D\left(\Delta^{+}, \widetilde{g}\right)^{-1} \sum_{w \in W(G(\mathbb{R}), H(\mathbb{R}))} \operatorname{sgn}(w) \frac{D\left(\Delta^{+}, w \widetilde{g}\right)}{\left|D\left(\Delta^{+}, w g\right)\right|} \frac{\left|D\left(\Psi_{i}, w g\right)\right|}{D\left(\Psi_{i}, \overline{w g}\right)} \Lambda_{M}(\overline{w g})
$$

The final terms equal

$$
\frac{e^{\rho}(w \widetilde{g}) / e^{\rho_{i}}(\overline{w g})}{\left|e^{\rho}(w \widetilde{g})\right| /\left|e^{\rho_{i}}(\overline{w g})\right|} \prod_{\Delta^{+} \backslash \Psi_{i}} \frac{\left(1-e^{-\alpha}(w g)\right)}{\left|\left(1-e^{-\alpha}(w g)\right)\right|} \Lambda_{M}(\overline{w g})
$$

Assume $\Delta^{+}$satisfies (2.15). Since $\theta \alpha=-\bar{\alpha}$, this says the positive complex roots come in pairs $\alpha, \bar{\alpha}$. Then the product becomes the product over $\Psi_{r}$, i.e. $\epsilon\left(\Psi_{r}, w g\right)$, giving

$$
\epsilon\left(\Psi_{r}, w g\right) \frac{e^{\rho}(w \widetilde{g}) / e^{\rho_{i}}(\overline{w g})}{\left|e^{\rho}(w \widetilde{g})\right| /\left|e^{\rho_{i}}(\overline{w g})\right|} \Lambda_{M}(\overline{w g})
$$

By Definition 4.1

$$
\Lambda_{M}(\overline{w g})=\left(\Lambda e^{-\rho}\right)(w g)\left(\gamma\left(\Psi_{r}\right)^{-1} e^{\rho_{r}}\right)(w g) e^{\rho_{c x}}(w g) e^{\rho_{i}}(\overline{w g})
$$


Using

$$
\begin{aligned}
\left(\Lambda e^{-\rho}\right)(w g) & =\Lambda(w \widetilde{g}) e^{-\rho}(w \widetilde{g}) \\
\left(\gamma\left(\Psi_{r}\right)^{-1} e^{\rho_{r}}\right)(w g) & =\left|e^{\rho_{r}}(w g)\right| \\
\left|e^{\rho}(w \widetilde{g})\right| /\left|e^{\rho_{i}}(\overline{w g})\right| & =\left|e^{\rho_{r}}(w g)\right| e^{\rho_{c x}}(w g) \quad \text { (since (2.15) holds) }
\end{aligned}
$$

it is easy to see the final two terms of (8.10) are equal to $\Lambda(w \widetilde{g})$. Therefore

$$
\Theta_{I(\gamma)}(g)=(-1)^{q_{H}} D\left(\Delta^{+}, \widetilde{g}\right)^{-1} \sum_{w \in W(G(\mathbb{R}), H(\mathbb{R}))} \operatorname{sgn}(w) \epsilon\left(\Psi_{r}, w g\right) \Lambda(w \widetilde{g}) .
$$

By Lemma 2.12(3) $\epsilon\left(\Psi_{r}, w g\right)=\operatorname{sgn}\left(w_{r}\right) \tau\left(\Psi_{r}, w_{r}^{-1}\right)(g) \epsilon\left(\Psi_{r}, g\right)$. Also (cf. Section 2$) \operatorname{sgn}(w) \operatorname{sgn}\left(w_{r}\right)=\operatorname{sgn}\left(w_{i}\right)$. Inserting these, and replacing $w$ with $w^{-1}$, gives (8.4).

This completes the proof provided $\Delta^{+}$satisfies $(2.15)$, i.e. $B\left(\Delta^{+}\right)=0$. The general case follows from Lemma 6.14.

Example 8.12 Consider principal series for $S L(2, \mathbb{R})$. We use the notation of Example 4.11.

Let $s_{\alpha} \in W$ be the simple reflection. Then $\tau\left(\Psi_{r}, s_{\alpha}\right)(h(x))=\operatorname{sgn}(\alpha(h(x)))=$ 1 , and (8.3) gives

$$
\begin{aligned}
\Theta_{I(\gamma)}(h(x)) & =\frac{\operatorname{sgn}\left(1-\frac{1}{x^{2}}\right)}{\left(1-\frac{1}{x^{2}}\right) x}\left(\Lambda(x)+\Lambda\left(x^{-1}\right)\right) \\
& =\frac{\operatorname{sgn}\left(x-\frac{1}{x}\right)}{x-\frac{1}{x}} \operatorname{sgn}(x)\left(\Lambda(x)+\Lambda\left(x^{-1}\right)\right) \\
& =\frac{(\Lambda \otimes \operatorname{sgn})(x)+(\Lambda \otimes \operatorname{sgn})\left(x^{-1}\right)}{\left|x-\frac{1}{x}\right|}
\end{aligned}
$$

This is the familiar character formula for $\operatorname{Ind}_{B(\mathbb{R})}^{S L(2, \mathbb{R})}(\Lambda \otimes \operatorname{sgn})(B(\mathbb{R})$ is a Borel subgroup). For example if $\Lambda=e^{\rho}$ then $e^{\rho}(h(x))=x$ and $(\Lambda \otimes \operatorname{sgn})(h(x))=$ $|x|$, so

$$
\theta_{I(\gamma)}(h(x))=\frac{|x|+|x|^{-1}}{\left|x-\frac{1}{x}\right|}
$$

This is the character formula of the spherical principal series with infinitesimal character $\rho$, with the trivial representation as a quotient. 
Example 8.15 Consider $P G L(2, \mathbb{R})$ and use notation of Example 4.12. We compute

$$
s_{\alpha}(h(x), z)=\left(h\left(\frac{1}{x}\right), e^{-2 \rho_{r}}(h(x)) z\right)=\left(h\left(\frac{1}{x}\right), \frac{z}{x}\right)=\left(h\left(\frac{1}{x}\right), \frac{1}{z}\right)
$$

and

$$
\tau\left(\Psi_{r}, s_{\alpha}\right)(h(x))=\operatorname{sgn}(\alpha(h(x)))=\operatorname{sgn}(x) .
$$

Write $\Lambda(h(x), z)=\mu(x) z$, so (8.3) gives

$$
\begin{aligned}
\Theta_{I(\gamma)}(h(x)) & =\Theta_{I(\gamma)}(h(x), z) \\
& =\frac{\operatorname{sgn}\left(1-\frac{1}{x}\right)}{\left(1-\frac{1}{x}\right) z}\left(\mu(x) z+\mu\left(x^{-1}\right) \operatorname{sgn}(x) / z\right) \\
& =\frac{\mu(x)+\mu\left(x^{-1}\right) \frac{1}{|x|}}{\left|1-\frac{1}{x}\right|} \\
& =\frac{\mu(x)|x|^{\frac{1}{2}}+\mu\left(x^{-1}\right)|x|^{-\frac{1}{2}}}{\left|1-\frac{1}{x}\right||x|^{\frac{1}{2}}}
\end{aligned}
$$

This is the formula for $\operatorname{Ind}_{B(\mathbb{R})}^{P G L(2, \mathbb{R})}\left(\left.\mu\right|^{\frac{1}{2}}\right)$. For example $\Lambda=e^{\rho}$ is given by $\mu=1$, and

$$
\theta_{I(\gamma)}(h(x))=\frac{|x|^{\frac{1}{2}}+|x|^{-\frac{1}{2}}}{\left|1-\frac{1}{x}\right||x|^{\frac{1}{2}}} .
$$

This is the spherical principal series with infinitesimal character $\rho$, with the trivial representation as a quotient. Note that $\theta_{I(\gamma)}(h(x))=1$ for $x<0$.

Example 8.18 This example generalizes (parts of) the previous two. Suppose $H(\mathbb{R})$ is split, and take $\Lambda=e^{\rho}$. Let $\pi=I(H, \Lambda)$. A short calculation gives

$$
\Theta_{I(\gamma)}(g)=\left|D\left(\Delta^{+}, g\right)\right|^{-1} \sum_{w \in W}\left|e^{w \rho}(g)\right| \quad(g \in H(\mathbb{R})) .
$$

This is the character of the spherical principal series representation with infinitesimal character $\rho$, with the trivial representation as quotient.

For later use we note a Corollary of the main Theorem. 
Corollary 8.20 Fix $H, \Delta^{+}$and $\Lambda$. Let $\gamma$ be standard limit data based on H. Then

$$
a\left(I(\gamma), \Delta^{+}, \Lambda\right)=\left\{\begin{array}{l}
(-1)^{q_{H}+B\left(\Delta^{+}\right)} \operatorname{sgn}(w) \quad \gamma \sim\left(H, w \Delta_{i}^{+}, \Delta_{r}^{+}, \Lambda\right) \quad\left(w \in W_{i}\right) \\
\text { otherwise. }
\end{array}\right.
$$

Proof. Write $\gamma=\left(H, \Psi_{i}, \Psi_{r}, \Gamma\right)$. Define $x \in W_{i}, y \in W_{r}$ by $x \Delta_{i}^{+}=$ $\Psi_{i}, y \Delta_{r}^{+}=\Psi_{r}$. Apply Lemma 6.10 or (6.6):

$$
a\left(I(\gamma), \Delta^{+}, \Lambda\right)=a\left(I(\gamma), x y \Delta^{+}, y \Lambda\right) \operatorname{sgn}(x)
$$

and we now have $\Psi_{i}, \Psi_{r} \subset x y \Delta^{+}$. By (8.4) this is non-zero if and only if $y \Lambda=\tau\left(\Psi_{r}, u\right) u \Gamma$ for some $u \in W(G(\mathbb{R}), H(\mathbb{R}))$, i.e. $\Gamma=\tau\left(\Psi_{r}, u^{-1}\right) u^{-1} y \Lambda$. So

$$
\begin{aligned}
\gamma=\left(H, \Psi_{i}, \Psi_{r}, \Gamma\right) & =\left(H, \Psi_{i}, \Psi_{r}, \tau\left(\Psi_{r}, u^{-1}\right) u^{-1} y \Lambda\right) \\
& \sim\left(H, \Psi_{i}, u^{-1} \Psi_{r}, u^{-1} y \Lambda\right) \quad \text { (by Definition 5.8) } \\
& \sim\left(H, u \Psi_{i}, \Psi_{r}, y \Lambda\right) \\
& \sim\left(H, u_{i} x \Delta_{i}^{+}, \Delta_{r}^{+}, \Lambda\right) .
\end{aligned}
$$

If this holds the sign is $(-1)^{q_{H}+B\left(\Delta^{+}\right)} \operatorname{sgn}\left(u_{i} x\right)$, so set $w=u_{i} x \in W_{i}$.

If $d \Lambda$ is regular with respect to the imaginary roots the statement may be simplified:

Corollary 8.24 In the setting of the previous Corollary, assume $\left\langle d \Lambda, \alpha^{\vee}\right\rangle \neq$ 0 for all imaginary roots. Then

$$
a\left(I(\gamma), \Delta^{+}, \Lambda\right)= \begin{cases}(-1)^{B\left(\Delta^{+}\right)}(-1)^{q_{H}} \epsilon\left(\Lambda, \Delta_{i}^{+}\right) & \gamma \sim\left(H, \Delta_{r}^{+}, \Lambda\right) \\ 0 & \text { otherwise. }\end{cases}
$$

In particular if $a\left(I(\gamma), \Delta^{+}, \Lambda\right) \neq 0$ and $a\left(I\left(\gamma^{\prime}\right), \Delta^{+}, \Lambda\right) \neq 0$ then $\gamma \sim \gamma^{\prime}$.

The conclusion of the Corollary does not hold if we drop the assumption that $\gamma$ is based on $H$. For example let $G=S L(2, \mathbb{R})$ and consider the principal series representations (cf. Example 8.12). The only principal series representation with the term $\Lambda(h(x))=x^{-1}$ is the spherical one. However this also occurs in the character formula for both of the discrete series representations (with trivial infinitesimal character), coming from the compact Cartan subgroup. We will see in the next section that such a result does hold for certain characters. See Section 14, Example 1. 


\section{Computation of $a\left(\pi, \Delta^{+}, \Lambda\right)$ for leading terms}

If $\Lambda$ satisfies a certain positivity condition with respect to $\Delta^{+}$then the conclusion of Corollary holds 8.20 without assuming $\gamma$ is based on $H$ :

Proposition 9.1 Fix $H, \Delta^{+}$and a genuine character $\Lambda$ of $H(\mathbb{R})_{\rho}$. Assume

(a) $\left\langle d \Lambda, \alpha^{\vee}\right\rangle \neq 0$ for all $\alpha \in \Delta$,

(b) $\operatorname{Re}\left\langle d \Lambda, \alpha^{\vee}\right\rangle \geq 0$ for all $\alpha \in \Delta_{r}^{+}$.

Suppose $\gamma$ is standard limit data. Then

$$
a\left(I(\gamma), \Delta^{+}, \Lambda\right)= \begin{cases}(-1)^{q_{H}+B\left(\Delta^{+}\right)} \epsilon\left(\Lambda, \Delta_{i}^{+}\right) & \gamma \sim\left(H, \Delta_{r}^{+}, \Lambda\right) \\ 0 & \text { otherwise. }\end{cases}
$$

The key point is that $a\left(I(\gamma), \Delta^{+}, \Lambda\right)=0$ if $\gamma$ is not based on $H$. If $I(\gamma)$ is a (relative) discrete series representation this follows from the fact that $I(\gamma)$ is tempered, and growth conditions on matrix coefficients [7]. For example see $[12,3.3]$ or $[15,13.26]$. The general case follows from this and the induced character formula. See [10, Section 3]. It also follows from the theory of $\mathcal{D}$-modules.

Corollary 9.3 Let $\pi$ be a virtual representation with regular infinitesimal character. Fix $H, \Delta^{+}$, and $\Lambda$ such that $\operatorname{Re}\left\langle d \Lambda, \alpha^{\vee}\right\rangle \geq 0$ for all $\alpha \in \Delta_{r}^{+}$. Then

$$
a\left(\pi, \Delta^{+}, \Lambda\right)=(-1)^{q_{H}+B\left(\Delta^{+}\right)} \epsilon\left(\Lambda, \Delta_{i}^{+}\right) M\left(I\left(H, \Delta_{r}^{+}, \Lambda\right), \pi\right) .
$$

\section{The Zuckerman Character formula for the Trivial Representation}

Recall

$$
\mathbb{C}=\sum_{\gamma} M(I(\gamma), \mathbb{C}) I(\gamma)
$$

for certain integers $M(I(\gamma), \mathbb{C})$. These are computed by the Kazhdan-LusztigVogan (KLV) polynomials, but in this case this goes back to a result of Zuckerman [21, Proposition 9.4.16]. We give an elementary proof here. 
Since we are working at regular integral infinitesimal character we may write $\gamma=(H, \Lambda)$ for a regular character and $I(H, \Lambda)$ for the corresponding standard module (cf. Section 4).

Fix $H$ and consider a standard module $I(H, \Lambda)$ with $\Lambda \in \mathcal{P}(H, \mathbb{C})$ (cf. (6.3)). Let

$$
\Delta^{+}=\Delta^{+}(\Lambda)=\left\{\alpha \mid\left\langle d \Lambda, \alpha^{\vee}\right\rangle>0\right\} .
$$

By definition $I(H, \Lambda)=I\left(H, \Delta_{r}^{+}, \Lambda\right)$ (see (5.7)). Therefore we may apply Corollary 9.3 to conclude

$$
M(I(H, \Lambda), \mathbb{C})=a\left(\mathbb{C}, \Delta^{+}(\Lambda), \Lambda\right)(-1)^{q_{H}+B\left(\Delta^{+}(\Lambda)\right)}
$$

(using $\epsilon\left(\Lambda, \Delta^{+}(\Lambda)_{i}\right)=1$ ). On the other hand by Corollary 7.3

$$
a\left(\mathbb{C}, \Delta^{+}(\Lambda), \Lambda\right)= \begin{cases}1 & \Lambda \in \mathcal{P}^{h o l}(H, \mathbb{C}) \\ 0 & \text { else }\end{cases}
$$

Therefore

$$
M(I(H, \Lambda), \mathbb{C})= \begin{cases}(-1)^{q_{H}+B\left(\Delta^{+}(\Lambda)\right)} & \Lambda \in \mathcal{P}^{\text {hol }}(H, \mathbb{C}) \\ 0 & \text { else. }\end{cases}
$$

In other words:

Lemma 10.6 There is an identity in the Grothendieck group

$$
\mathbb{C}=\sum_{H} \sum_{\Lambda \in \mathcal{P}_{\text {hol }}(H, \mathbb{C})}(-1)^{q_{H}+B\left(\Delta^{+}(\Lambda)\right)} I(H, \Lambda) .
$$

(the first sum is over $G(\mathbb{R})$-conjugacy classes of Cartan subgroups defined over $\mathbb{R})$.

Subtracting (a) and (c) in [21, Lemma 9.4.15] shows that

$$
(-1)^{q_{H}+B\left(\Delta^{+}(\Lambda)\right)}=(-1)^{\ell\left(\gamma_{0}\right)-\ell(\gamma)}
$$

where $\ell$ is the length function of [21, Definition 8.1.4] and $\gamma_{0}, \gamma$ are the parameters for the trivial representation and $I(H, \Lambda)$, respectively. Thus the Lemma is a version of Zuckerman's character formula for the trivial representation [21, Proposition 9.4.16]. A nice feature of these parameters is that it is precisely the holomorphic characters which appear. 


\section{Coherent Continuation}

We give the definition of coherent continuation [10, (3.38)], [21, Definitions $7.2 .5,7.2 .28]$ in our terms.

Fix a Cartan subgroup $H$, with Weyl group $W$, and $\lambda \in \mathfrak{h}^{*}$. Let $X^{*}(H)$ be the algebraic (holomorphic) characters of $H$. If $F$ is a finite dimensional representation of $G(\mathbb{C})$ write $\Delta(F) \subset X^{*}(H)$ for its weights.

We say a family $\left\{\pi[\lambda+\mu] \mid \mu \in X^{*}(H)\right\}$ of virtual Harish-Chandra modules is a coherent family if for all $\mu \in X^{*}(H)$ :

(1) $\pi[\lambda+\mu]$ has infinitesimal character $\lambda+\mu$,

(2) If $F$ is any finite dimensional representation of $G(\mathbb{C})$ then

$$
\pi[\lambda+\mu] \otimes F=\sum_{\mu^{\prime} \in \Delta(F)} \pi\left[\lambda+\mu+\mu^{\prime}\right]
$$

where $\Delta(F)$ is the set of weights of $F$.

We consider only finite dimensional representations of $G(\mathbb{C})$ rather than of $G(\mathbb{R})$ as in [21, Chapter 7] - this is sufficient for our purposes.

This has a direct interpretation in terms of characters. Fix $\Delta^{+}$and drop it from the notation, so write $a(\pi[\lambda+\mu], \Lambda)$ for the coefficients in the character formula for $\pi[\lambda+\mu]$.

Lemma 11.1 (a) Suppose $\pi$ is a virtual character with regular infinitesimal character $\lambda$. There is a unique coherent family $\left.\left\{\pi[\lambda+\mu] \mid \mu \in X^{*}(H)\right]\right\}$ such that $\pi[\lambda]=\pi$.

(b) Suppose $\{\pi[\lambda+\mu]\}$ is a coherent family. Assume $d \Lambda=w \lambda$ for some $w \in W$, and $\mu \in X^{*}(H)$. Then

$$
a(\pi[\lambda+\mu], \Lambda \otimes w \mu)=a(\pi[\lambda], \Lambda) .
$$

For (a) see [10, Lemma 3.39] or [21, Theorem 7.2.7 and Corollary 7.2.27]. Part (b) is the first statement of [10, Lemma 3.44] carried over to our setting. This can be made into a necessary condition by considering all Cartan subgroups, but we won't need this.

Lemma 11.3 Suppose $\{\pi[\lambda+\mu]\}$ is a coherent family, and fix $w \in W(\lambda)$. Suppose $d \Lambda \in W \lambda$, and write $d \Lambda=y w \lambda$ for $y \in W$. Then

$$
a(\pi[w \lambda], \Lambda)=a\left(\pi[\lambda],\left(y w^{-1} y^{-1}\right) \times \Lambda\right) .
$$


Proof. This is a simple change of variables. Let $\mu=w \lambda-\lambda$ and consider $\Lambda \otimes(-y \mu)$. Then $d(\Lambda \otimes(-y \mu))=y w \lambda-y \mu=y(w \lambda-\mu)=y \lambda$. Apply (11.2):

$$
\begin{aligned}
a(\pi[w \lambda], \Lambda) & =a(\pi[\lambda+\mu],(\Lambda \otimes(-y \mu)) \otimes y \mu) \\
& =a(\pi[\lambda], \Lambda \otimes(-y \mu)) .
\end{aligned}
$$

It is enough to show $\left(y w^{-1} y^{-1}\right) \times \Lambda=\Lambda-y \mu$, i.e. $\left(y w^{-1} y^{-1}\right) d \Lambda-d \Lambda=-y \mu$. This follows from $d \Lambda=y w \lambda$ and $\lambda-w \lambda=-\mu$.

Fix once and for all an abstract Cartan subalgebra $\mathfrak{h}_{a}$, a choice of positive roots $\Delta_{a}^{+}$of $\mathfrak{h}_{a}$, and let $W_{a}$ be the abstract Weyl group, as in $[22,2.6]$. If $\mathfrak{h}$ is any Cartan subalgebra, and $\lambda \in \mathfrak{h}^{*}$, there is a unique $\lambda_{a} \in \mathfrak{h}_{a}^{*}$, dominant for $\Delta_{a}^{+}$, and a unique inner isomorphism $\phi_{\lambda}$ taking $\lambda_{a}$ to $\lambda$. This induces an isomorphism $\phi_{\lambda}: W_{a} \rightarrow W$. We need an identity describing the dependence on $\lambda$, which is immediate:

$$
\phi_{y \lambda}\left(w_{a}\right)=y \phi_{\lambda}\left(w_{a}\right) y^{-1} \quad\left(y \in W, w_{a} \in W_{a}\right) .
$$

Definition 11.7 Suppose $\pi$ is a virtual character, with regular infinitesimal character $\lambda_{a} \in \mathfrak{h}_{a}^{*}$ (dominant for $\Delta_{a}^{+}$), and $w_{a} \in W_{a}\left(\lambda_{a}\right)$. Fix a Cartan subgroup $H$ and $\lambda \in \mathfrak{h}^{*}$ conjugate to $\lambda_{a}$. Let $\{\pi[\lambda+\gamma]\}$ be the coherent family with $\pi=\pi[\lambda]$.

For $w_{a} \in W_{a}\left(\lambda_{a}\right)$ define

$$
w_{a} \cdot \pi=\pi\left[\phi_{\lambda}\left(w_{a}^{-1}\right) \lambda\right] .
$$

This is independent of the choice of $H$ and $\lambda$.

This is the coherent continuation action on virtual characters, due to Zuckerman. See [21, Definition 7.2.28].

Here is the formulation in terms of characters. We first define the cross action of the abstract Weyl group on genuine characters (cf. (11.9)). For $w_{a} \in W\left(\lambda_{a}\right)$, and $\Lambda$ such that $d \Lambda$ is conjugate to $\lambda_{a}$, define

$$
w_{a} \times \Lambda=\phi_{d \Lambda}\left(w_{a}^{-1}\right) \times \Lambda .
$$

Note that the differential of the right hand side is $d\left(w_{a}^{-1} \times \Lambda\right)=\phi_{d \Lambda}\left(w_{a} \lambda_{a}\right)$, and this implies $d\left(w_{a} \times \Lambda\right)-d \Lambda=\phi_{d \Lambda}\left(w_{a}^{-1} \lambda_{a}-\lambda_{a}\right)$, which is a sum of roots since $w_{a} \in W\left(\lambda_{a}\right)$, so the cross action is well defined. It is straightforward to check that $\left(x_{a} y_{a}\right) \times \Lambda=x_{a} \times\left(y_{a} \times \Lambda\right)$. 
Proposition 11.10 Suppose $\pi$ is a virtual character, with regular infinitesimal character $\lambda_{a} \in \mathfrak{h}_{a}^{*}$ (dominant for $\Delta_{a}^{+}$), and $w_{a} \in W_{a}\left(\lambda_{a}\right)$. Suppose $H$ is a Cartan subgroup, defined over $\mathbb{R}$, and $\Lambda$ is a genuine character of $\widetilde{H(\mathbb{R})}$ with $d \Lambda$ conjugate to $\lambda_{a}$. Then

$$
a\left(w_{a} \cdot \pi, \Lambda\right)=a\left(\pi, w_{a}^{-1} \times \Lambda\right) .
$$

Proof. This is a straightforward unwinding of the definitions. First of all choose $\lambda \in \mathfrak{h}^{*}$ conjugate to $\lambda_{a}$. Let $\{\pi[\lambda+\mu]\}$ be the coherent family with $\pi[\lambda]=\pi$. Then

$$
\begin{aligned}
a\left(w_{a} \cdot \pi, \Lambda\right) & =a\left(w_{a} \cdot \pi[\lambda], \Lambda\right) \\
& =a\left(\pi\left[\phi_{\lambda}\left(w_{a}^{-1}\right) \lambda\right], \Lambda\right) \quad(\text { by Definition 11.7) } \\
& =a\left(\pi,\left(y \phi_{\lambda}\left(w_{a}\right) y^{-1}\right) \times \Lambda\right) \quad(\text { by }(11.4))
\end{aligned}
$$

where $y \in W$ satisfies $y \phi_{\lambda}\left(w_{a}^{-1}\right) \lambda=d \Lambda$.

On the other hand

$$
\begin{aligned}
a\left(\pi, w_{a}^{-1} \times \Lambda\right) & =a\left(\pi, \phi_{d \Lambda}\left(w_{a}\right) \times \Lambda\right) \\
& =a\left(\pi, \phi_{y \phi_{\lambda}\left(w_{a}^{-1}\right) \lambda}\left(w_{a}\right) \times \Lambda\right. \\
& =a\left(\pi,\left(y \phi_{\lambda}\left(w_{a}^{-1}\right) \phi_{\lambda}\left(w_{a}\right) \phi_{\lambda}\left(w_{a}\right) y^{-1}\right) \times \Lambda\right) \quad(\text { by }(11.6)) \\
& =a\left(\pi,\left(y \phi_{\lambda}\left(w_{a}\right) y^{-1}\right) \times \Lambda\right)
\end{aligned}
$$

Remark 11.12 Note that for $w_{a} \in W_{a} w_{a} \times \Lambda=w \times \Lambda$ for some $w \in W$ depending on $\Lambda$, so some care is needed when using this formula when $\Lambda$ varies. Fix $\Lambda_{0}$, and let $w_{0}=\phi_{d \Lambda_{0}}\left(w_{a}^{-1}\right) \in W$, so

$$
w_{a}^{-1} \times \Lambda=w_{0}^{-1} \times \Lambda \quad\left(\text { if } d \Lambda_{0}=d \Lambda\right) .
$$

Then

$$
w_{a}^{-1} \times \Lambda=\left(y w_{0}^{-1} y^{-1}\right) \times \Lambda \quad\left(\text { if } y d \Lambda_{0}=d \Lambda\right) .
$$

Example 11.14 Consider the trivial representation $\mathbb{C}$ :

$$
w_{a} \cdot \mathbb{C}=\operatorname{sgn}\left(w_{a}\right) \mathbb{C} \quad\left(w_{a} \in W_{a}\right) .
$$

This is immediate from (7.5) and (11.11). 


\section{Computation of general $a\left(\pi, \Delta^{+}, \Lambda\right)$}

As in the previous section fix $\mathfrak{h}_{a}, W_{a}$, and define the cross action of $W_{a}$ accordingly. We use coherent continuation to give a formula for $a\left(\pi, \Delta^{+}, \Lambda\right)$ for arbitrary $\Lambda$.

Proposition 12.1 Let $\pi$ be a virtual representation with regular infinitesimal character $\lambda_{a} \in \mathfrak{h}_{a}^{*}$. Fix $H, \Delta^{+}$and a genuine character $\Lambda$ of $H(\mathbb{R})_{\rho}$ with $d \Lambda$ conjugate to $\lambda_{a}$. Suppose $w_{a} \in W_{a}\left(\lambda_{a}\right)$ satisfies

$$
\operatorname{Re}\left\langle d\left(w_{a} \times \Lambda\right), \alpha^{\vee}\right\rangle \geq 0 \text { for all } \alpha \in \Delta_{r}^{+} .
$$

Then

$$
a\left(\pi, \Delta^{+}, \Lambda\right)=(-1)^{q_{H}+B\left(\Delta^{+}\right)} \epsilon\left(w_{a} \times \Lambda, \Delta_{i}^{+}\right) M\left(I\left(H, \Delta_{r}^{+}, w_{a} \times \Lambda\right), w_{a} \cdot \pi\right) .
$$

Proof. By Propositions 11.10 and Corollary 9.3

$$
\begin{aligned}
a\left(\pi, \Delta^{+}, \Lambda\right) & =a\left(w_{a} \cdot \pi, \Delta^{+}, w_{a} \times \Lambda\right) \\
& =(-1)^{q_{H}+B\left(\Delta^{+}\right)} \epsilon\left(w_{a} \times \Lambda, \Delta_{i}^{+}\right) M\left(I\left(H, \Delta_{r}^{+}, w_{a} \times \Lambda\right), w_{a} \cdot \pi\right) .
\end{aligned}
$$

If $\lambda_{a}$ is integral we can always find $w_{a} \in W_{a}\left(\lambda_{a}\right)=W_{a}$ satisfying the conditions, so this determines all coefficients. In fact there is some flexibility in choosing $w_{a}$. If $\lambda_{a}$ is not integral a little more work is required to determine all coefficients.

Here is one convenient reformulation in the integral case.

Corollary 12.4 Assume $\lambda_{a}$ is regular and integral. Suppose $\pi$ is a virtual character with infinitesimal character $\lambda_{a}$, and having a central character. Choose $\Delta^{+}$so that (2.15) holds, and let $\lambda$ be $\Delta^{+}$-dominant and conjugate to $\lambda_{a}$. Let $\Lambda_{1}, \ldots, \Lambda_{n}$ be the genuine characters of $\widehat{H(\mathbb{R})}$ such that $d \Lambda_{i}=\lambda$, and such that $\Lambda_{i} e^{-\rho}$ and $\pi$ have the same restriction to $Z(G(\mathbb{R}))$.

Then for any $w_{a} \in W_{a}$ and $i \leq n$ :

$$
a\left(\pi, \Delta^{+}, w_{a}^{-1} \times \Lambda_{i}\right)=(-1)^{q_{H}} M\left(I\left(H, \Lambda_{i}\right), w_{a} \cdot \pi\right) .
$$

Every nonzero $a\left(\pi, \Delta^{+}, \Lambda\right)$ is of this form. 
We now sketch how to use this to compute $a\left(\pi, \Delta^{+}, \Gamma\right)$ using the atlas software, which is freely available from www.liegroups.org/software. We assume the infinitesimal character is regular and integral.

First assume $I(\gamma)$ is a standard module and $H$ is an arbitrary $(\theta$-stable) Cartan subgroup. With $\Delta^{+}$and $\Lambda_{i}$ as in Corollary 12.4 we conclude

$$
a\left(I(\gamma), \Delta^{+}, w_{a}^{-1} \times \Lambda_{i}\right)=(-1)^{q_{H}} M\left(I\left(H, \Lambda_{i}\right), w_{a} \cdot I(\gamma)\right) .
$$

The list of parameters with regular integral infinitesimal character is given by the output of the block command in the atlas software. First identify the modules $I\left(H, \Lambda_{i}\right)$ for $1 \leq i \leq n$ in the output of block.

Next compute $w_{a} \cdot I(\gamma)$ for all $w_{a} \in W_{a}$ in the basis of standard modules. This is elementary; it requires only the output of the block command, and not the KLV polynomials. In particular if $s_{\alpha}$ is a simple reflection then $s_{\alpha} \cdot I(\gamma)$ is a single standard module unless $\alpha$ is noncompact imaginary, in which case it is given by a Hecht-Schmid identity with 2 or 3 terms. See [9], [21, Corollary 8.4.6] or [23]. The application coherentContinuation, available at www. liegroups.org/software/helpers, is useful for computing coherent continuation. See [2].

Then (12.6) gives all nonzero terms $a\left(I(\gamma), \Delta^{+}, \Lambda\right)$. To vary $\Delta^{+}$use Lemma 6.10. This can be used to compute the character of a discrete series representation on an arbitrary Cartan subgroup.

For example for the split real form of $E_{8}$, it take about 14 seconds on a small computer to compute the block command, which produces a list of 452, 690 standard modules. The output of this command takes up about 165 megabytes of disk space.

Now suppose $\pi$ is an irreducible representation. There are at least two ways to compute $a\left(\pi, \Delta^{+}, \Lambda\right)$. First of all use the KLV polynomials to write $\pi$ as a sum of standard modules

$$
\pi=\sum_{\gamma} M(I(\gamma), \pi) I(\gamma)
$$

Then

$$
a\left(\pi, \Delta^{+}, \Lambda\right)=\sum_{\gamma} M(I(\gamma), \pi) a\left(I(\gamma), \Delta^{+}, \Lambda\right)
$$

and $a\left(I(\gamma), \Delta^{+}, \Lambda\right)$ are computed as above. The KLV polynomials, and hence $M(I(\gamma), \pi)$, are provided by the klbasis command of atlas. These are 
readily available for groups of rank $\leq 7$. However, in contrast with the block command, these are difficult to compute in higher rank. For example to compute the KLV polynomials for the split real form of $E_{8}$ takes about 5.5 hours on a very large machine, and storing them takes 60 gigabytes of disk space.

Alternatively, the wgraph command of atlas computes the coherent continuation action in the basis of irreducible modules. Note, however, that computing the wgraph command is the same order of difficulty as computing klbasis. In any event one can compute

$$
a\left(\pi, \Delta^{+}, w_{a}^{-1} \times \Lambda_{i}\right)=(-1)^{q_{H}} M\left(I\left(H, \Lambda_{i}\right), w_{a} \cdot \pi\right)
$$

directly, although this uses the KLV polynomials twice, once to compute $w_{a} \cdot \pi$, and again to compute $M\left(I\left(H, \Lambda_{i}\right), w_{a} \cdot \pi\right)$.

\section{Alternative version}

While the parametrization of Section 5 using the $\rho$-cover of $H$ has many advantages, the extra choice of $\Psi_{r}$ is unappealing. For this reason it worthwhile to write Langlands parameters in terms of the $\rho_{i}$ cover of $H$. This is done in [1]. It is useful to give the translation between the two versions.

Definition $13.1 \rho_{i}$-standard limit data is a triple $\left(H, \Psi_{i}, \Gamma\right)$ where $H$ is a Cartan subgroup (defined over $\mathbb{R}$ as usual), $\Psi_{i}$ is a set of positive imaginary roots, and $\Gamma$ is a genuine character of $H(\mathbb{R})_{\rho_{i}}$ (where $\rho_{i}$ is defined by $\Delta_{i}^{+}$). We assume these satisfy

$$
\begin{array}{ll}
\left\langle d \Gamma, \alpha^{\vee}\right\rangle \geq 0 & \left(\alpha \in \Psi_{i}\right) \\
\left\langle d \Gamma, \alpha^{\vee}\right\rangle \neq 0 & \left(\alpha \in \Psi_{i} \text { simple and compact }\right)
\end{array}
$$

We define equivalence by conjugation by $G(\mathbb{R})$.

See [1, Theorem 6.1].

Lemma 13.4 Suppose $\left(H, \Psi_{i}, \Psi_{r}, \Lambda\right)$ is standard limit data. Define $\rho_{i}$-standard limit data $\left(H, \Psi_{i}, \Lambda \otimes \gamma\left(\Psi_{r}\right)^{-1}\right)$ (see Section 4).

Conversely suppose $\left(H, \Psi_{i}, \Gamma\right)$ is $\rho_{i}$-standard limit data. Choose a set of positive roots $\Psi_{r}$, and define standard limit data $\left(H, \Psi_{i}, \Psi_{r}, \Gamma \otimes \gamma\left(\Psi_{r}\right)\right)$. 
These define a bijection between equivalence classes of standard limit data and $\rho_{i}$-standard limit data.

The proof is immediate (using (4.4)).

To write a character formula using $\rho_{i}$-standard limit data we need a version of the Weyl denominator defined on $H(\mathbb{R})_{\rho_{i}}$.

Definition 13.5 Fix a set $\Delta^{+}$of positive roots, and define $\rho_{i}, \rho_{r}$ and $\rho_{c x}$ as usual. Recall (end of Section 2) $e^{\rho_{c x}}$ is well defined on $H(\mathbb{R})$. Define a genuine character $e^{\rho^{\prime}}$ of $H(\mathbb{R})_{\rho_{i}}$ :

$$
e^{\rho^{\prime}}(\bar{g})=e^{\rho_{c x}}(g)\left|e^{\rho_{r}}(g)\right| e^{\rho_{i}}(\bar{g}) \quad\left(g \in H(\mathbb{R})_{\rho_{i}}\right)
$$

where $g$ is the image of $\bar{g}$ in $H(\mathbb{R})$, and

$$
D^{\prime}\left(\Delta^{+}, \bar{g}\right)=\prod_{\alpha \in \Delta^{+}}\left(1-e^{-\alpha}(g)\right) e^{\rho^{\prime}}(\bar{g})
$$

We then define constants $a^{\prime}\left(\pi, \Delta^{+}, \Lambda\right)$ for $\Lambda$ a genuine character of $H(\mathbb{R})_{\rho_{i}}$ by analogy with (6.5), using $D^{\prime}$ in place of $D$.

It follows easily from Definition (4.1) that

$$
e^{\rho^{\prime}}=e^{\rho} \otimes \gamma\left(\Delta_{r}^{+}\right)^{-1}
$$

Suppose $\Lambda$ is a genuine character of $H(\mathbb{R})_{\rho}$. It follows immediately from this and Definition (4.3) that

$$
\left(\Lambda e^{-\rho}\right)(g)=\left(\Lambda_{M} e^{-\rho^{\prime}}\right)(g)
$$

Also an elementary calculation gives

$$
(w \Lambda)_{M}=\tau\left(\Psi_{r}, w\right) \Lambda_{M} \quad(w \in W(G(\mathbb{R}), H(\mathbb{R})) .
$$

The character formula of Proposition 8.3 takes the following form.

Proposition 13.11 Suppose $\gamma=\left(H, \Psi_{i}, \Lambda\right)$ is $\rho_{i}$-standard limit data. Choose positive roots $\Delta^{+}$containing $\Psi_{i}$, and let $\Psi_{r}=\Delta_{r}^{+}$. Let $\delta=(-1)^{q_{H}+B\left(\Delta^{+}\right)}$. Then for $g \in H(\mathbb{R})_{\text {reg }}$

$$
\Theta_{I(\gamma)}(g)=\delta \frac{\epsilon\left(\Psi_{r}, g\right)}{D^{\prime}\left(\Delta^{+}, \bar{g}\right)} \sum_{w \in W(G(\mathbb{R}), H(\mathbb{R}))} \operatorname{sgn}_{i}(w)(w \Lambda)(\bar{g}) .
$$


where $\bar{g}$ is an inverse image of $g$ in $H(\mathbb{R})_{\rho_{i}}$. In other words for $\Gamma$ a genuine character of $H(\mathbb{R})_{\rho_{i}}$ :

$$
a^{\prime}\left(I(\gamma), \Delta^{+}, \Gamma\right)=\left\{\begin{array}{ll}
\delta \operatorname{sgn}_{i}(w) & \Gamma=w \Lambda \quad \text { otherwise }
\end{array} \quad(w \in W(G(\mathbb{R}), H(\mathbb{R}))\right.
$$

The remaining results, including Proposition 12.1, hold with minor changes.

\section{Examples}

We compute some character formulas for $S L(2, \mathbb{R}), P G L(2, \mathbb{R})$ and $S p(4, \mathbb{R})$, using Corollary (12.4).

\subsection{Example 1: $S L(2, \mathbb{R})$}

Here is the character table for $S L(2, \mathbb{R})$ at infinitesimal character $\rho$. See Examples 4.11 and 8.12.

Since $\rho$ exponentiates to $H$ we may ignore the $\rho$-cover. Identify compact and split Cartan subgroup with $\mathbb{R}^{*}, S^{1}$ respectively. Choose $\Delta^{+}=\{\alpha\}$ for $\mathbb{R}^{*}$ with $\alpha(x)=x^{2}$, and for $S^{1}$ with $\alpha\left(e^{i \theta}\right)=e^{2 i \theta}$.

With the obvious notation there are 4 standard modules:

$$
\begin{aligned}
D S_{+}=I\left(S^{1}, e^{i \theta}\right) & : \text { holomorphic discrete series } \\
D S_{-}=I\left(S^{1}, e^{-i \theta}\right) & : \text { anti-holomorphic discrete series } \\
P S_{+}=I\left(\mathbb{R}^{*}, x\right) & : \text { reducible principal series with even } K \text {-types } \\
P S_{-}=I\left(\mathbb{R}^{*},|x|\right) & : \text { irreducible principal series with odd } K \text {-types }
\end{aligned}
$$

The only reducible standard module is $P S_{+}$; in the Grothendieck group

$$
P S_{+}=D S_{+}+D S_{-}+\mathbb{C}
$$

Since $\Delta^{+}$is fixed we drop it from the notation. The coefficients $a(I(\gamma), \Lambda)$ and $a(\pi, \Lambda)$ are given by the following table. 


\begin{tabular}{|c|cccccc|}
\hline \multicolumn{7}{|c|}{$S L(2, \mathbb{R})$} \\
\hline & \multicolumn{7}{|c|}{$\mathbb{R}^{*}$} & \multicolumn{3}{c|}{$S^{1}$} \\
\hline & $x$ & $|x|$ & $\frac{1}{x}$ & $\frac{1}{|x|}$ & $e^{i \theta}$ & $e^{-i \theta}$ \\
\hline$D S_{+}$ & 0 & 0 & 1 & 0 & -1 & 0 \\
\hline$D S_{-}$ & 0 & 0 & 1 & 0 & 0 & 1 \\
\hline$P S_{+}$ & 1 & 0 & 1 & 0 & 0 & 0 \\
\hline$P S_{-}$ & 0 & 1 & 0 & 1 & 0 & 0 \\
\hline \hline $\mathbb{C}$ & 1 & 0 & -1 & 0 & 1 & -1 \\
\hline
\end{tabular}

We show how to compute some of these coefficients. The main coherent continuation identity we need is the basic Hecht-Schmid identity:

$$
s_{\alpha} \cdot D S_{ \pm}=P S_{+}-D S_{\mp}
$$

and we'll also use

$$
s_{\alpha} \cdot P S_{ \pm}=P S_{ \pm}
$$

which follows from (a) and (14.2).

First we consider the easy character formulas for $P S_{+}$on $H=\mathbb{R}^{*}$. Using (12.5), with $q_{H}=0=B\left(\Delta^{+}\right)=0$, we have:

$$
\begin{aligned}
a\left(P S_{+}, x\right) & =M\left(I(H, x), P S_{+}\right)=M\left(P S_{+}, P S_{+}\right)=1 \\
a\left(P S_{+},|x|\right) & =M\left(I(H,|x|), P S_{+}\right)=M\left(P S_{-}, P S_{+}\right)=0 \\
a\left(P S_{+}, x^{-1}\right) & =a\left(P S_{+}, s_{\alpha} \times x\right)=M\left(I(H, x), s_{\alpha} \cdot P S_{+}\right)=M\left(P S_{+}, P S_{+}\right)=1 \\
a\left(P S_{+},|x|^{-1}\right) & =a\left(P S_{+}, s_{\alpha} \times|x|\right)=M\left(I(H,|x|), s_{\alpha} \cdot P S_{+}\right)=M\left(P S_{-}, P S_{+}\right)=0
\end{aligned}
$$

The formulas for the characters of the principal series are given in Example 8.12 .

Next, here are the elementary character formulas for $D S_{ \pm}$on $H=S^{1}$. In this case $q_{H}=1$ and $B\left(\Delta^{+}\right)=0$. Using $I\left(H, e^{i \theta}\right)=D S_{+}$and (12.5) we compute:

$$
\begin{aligned}
a\left(D S_{+}, e^{i \theta}\right) & =-M\left(D S_{+}, D S_{+}\right)=-1 \\
a\left(D S_{+}, e^{-i \theta}\right) & =a\left(D S_{+}, s_{\alpha} \times e^{i \theta}\right)=-M\left(D S_{+}, s_{\alpha} \cdot D S_{+}\right) \\
& =-M\left(D S_{+}, P S-D S_{-}\right)=0 \\
a\left(D S_{-}, e^{i \theta}\right) & =-M\left(D S_{+}, D S_{-}\right)=0 \\
a\left(D S_{-}, e^{-i \theta}\right) & =a\left(D S_{-}, s_{\alpha} \times e^{i \theta}\right)=-M\left(D S_{+}, s_{\alpha} \cdot D S_{-}\right) \\
& =-M\left(D S_{+}, P S-D S_{+}\right)=1
\end{aligned}
$$


These give the well known formulas (and special cases of (3.3)):

$$
\begin{aligned}
& \theta_{D S_{+}}\left(e^{i \theta}\right)=\frac{-e^{i \theta}}{e^{i \theta}-e^{-i \theta}} \\
& \theta_{D S_{-}}\left(e^{i \theta}\right)=\frac{e^{-i \theta}}{e^{i \theta}-e^{-i \theta}}
\end{aligned}
$$

Finally the most interesting case, the discrete series characters on $\mathbb{R}^{*}$ :

$$
\begin{aligned}
a\left(D S_{ \pm}, x\right) & =M\left(I\left(\mathbb{R}^{*}, x\right), D S_{ \pm}\right)=M\left(P S_{+}, D S_{ \pm}\right)=0 \\
a\left(D S_{ \pm},|x|\right) & =M\left(I\left(\mathbb{R}^{*},|x|\right), D S_{ \pm}\right)=M\left(P S_{-}, D S_{ \pm}\right)=0 \\
a\left(D S_{ \pm}, \frac{1}{x}\right) & =a\left(D S_{ \pm}, s_{\alpha} \times x\right)=M\left(I\left(\mathbb{R}^{*}, x\right), s_{\alpha} \cdot D S_{ \pm}\right) \\
& =M\left(P S_{+}, P S_{+}-D S_{\mp}\right)=1 \\
a\left(D S_{ \pm}, \frac{1}{|x|}\right) & =a\left(D S_{ \pm}, s_{\alpha} \times|x|\right)=M\left(I\left(\mathbb{R}^{*},|x|\right), s_{\alpha} \cdot D S_{ \pm}\right) \\
& =M\left(P S_{-}, P S_{+}-D S_{\mp}\right)=0
\end{aligned}
$$

Therefore

$$
\theta_{D S_{ \pm}}(x)=\frac{x^{-1}}{x-\frac{1}{x}} \quad(|x|>1)
$$

This implies

$$
\theta_{D S_{ \pm}}(x)=\frac{-x}{x-\frac{1}{x}} \quad(|x|<1)
$$

\section{$14.2 \quad$ Example 2: $P G L(2, \mathbb{R})$}

We now give the character table for $P G L(2, \mathbb{R})$ at infinitesimal character $\rho$. We cannot ignore the $\rho$-cover in this case. See Examples 4.12 and 8.15.

Again identify compact and split Cartan subgroupos with $\mathbb{R}^{*}, S^{1}$ respectively. Choose $\Delta^{+}=\{\alpha\}$ for $\mathbb{R}^{*}$ with $\alpha(x)=x$, and for $S^{1}$ with $\alpha\left(e^{i \theta}\right)=e^{i \theta}$.

Now the cover of $\mathbb{R}^{*}$ is $\left\{(x, z) \mid z^{2}=x\right\}$, and $e^{\rho}(x, z)=z$. Similarly the cover of $S^{1}$ is $\left\{(w, z)|| w|=| z \mid=1, z^{2}=w\right\}$, and $e^{\rho}(w, z)=z$.

There are 3 standard modules at infinitesimal character $\rho$ :

$$
\begin{gathered}
D S=I\left(S^{1}, e^{\rho}\right): \text { discrete series } \\
P S_{\mathbb{C}}=I\left(\mathbb{R}^{*}, e^{\rho}\right): \text { reducible spherical principal series } \\
P S_{\text {sgn }}=I\left(\mathbb{R}^{*}, \operatorname{sgn} e^{\rho}\right): \text { reducible non-spherical principal series }
\end{gathered}
$$


Both standard modules are reducible; in the Grothendieck group

$$
\begin{aligned}
P S_{\mathbb{C}} & =D S+\mathbb{C} \\
P S_{\mathrm{sgn}} & =D S+\mathrm{sgn}
\end{aligned}
$$

There is no irreducible principal series representation at infinitesimal character $\rho$ (there are two irreducible principal series at infinitesimal character $2 \rho$, which we don't consider).

The coefficients $a(I(\gamma), \Lambda)$ and $a(\pi, \Lambda)$ are given by the following table.

\begin{tabular}{|c|cccccc|}
\hline \multicolumn{7}{|c|}{$P G L(2, \mathbb{R})$} \\
\hline & \multicolumn{7}{|c|}{$\mathbb{R}^{*}$} & \multicolumn{2}{c|}{$S^{1}$} \\
\hline & $e^{\rho}$ & $\operatorname{sgn} e^{\rho}$ & $e^{-\rho}$ & $\operatorname{sgn} e^{-\rho}$ & $e^{\rho}$ & $e^{-\rho}$ \\
\hline$D S$ & 0 & 0 & 1 & 1 & -1 & 1 \\
\hline$P S_{\mathbb{C}}$ & 1 & 0 & 0 & 1 & 0 & 0 \\
\hline$P S_{\text {sgn }}$ & 0 & 1 & 1 & 0 & 0 & 0 \\
\hline \hline $\mathbb{C}$ & 1 & 0 & -1 & 0 & 1 & -1 \\
\hline $\operatorname{sgn}$ & 0 & 1 & 0 & -1 & 1 & -1 \\
\hline
\end{tabular}

In this case the relevant Hecht-Schmid identity is:

$$
s_{\alpha} \cdot D S=P S_{\mathbb{C}}+P S_{\mathrm{sgn}}-D S
$$

We also use that fact that

$$
s_{\alpha} \cdot P S_{\mathbb{C}}=P S_{\mathrm{sgn}}
$$

which follows from (a), (14.8)(a) and Example 11.14.

Here is the easy character formula for $P S_{\mathbb{C}}$ on $H=\mathbb{R}^{*}$. In this case $q_{H}=1$ and $B\left(\Delta^{+}\right)=0$.

$$
\begin{aligned}
a\left(P S_{\mathbb{C}}, e^{\rho}\right) & =M\left(I\left(H, e^{\rho}\right), P S_{\mathbb{C}}\right)=M\left(P S_{\mathbb{C}}, P S_{\mathbb{C}}\right)=1 \\
a\left(P S_{\mathbb{C}}, \operatorname{sgn} e^{\rho}\right) & =M\left(I\left(H, e^{\rho} \operatorname{sgn}\right), P S_{\mathbb{C}}\right)=M\left(P S_{\mathrm{sgn}}, P S_{\mathbb{C}}\right)=0 \\
a\left(P S_{\mathbb{C}}, e^{-\rho}\right) & =a\left(P S_{\mathbb{C}}, s_{\alpha} \times e^{\rho}\right)=M\left(I\left(H, e^{\rho}\right), s_{\alpha} \cdot P S_{\mathbb{C}}\right) \\
& =M\left(P S_{\mathbb{C}}, P S_{\mathrm{sgn}}\right)=0 \\
a\left(P S_{\mathbb{C}}, \operatorname{sgn} e^{-\rho}\right) & =a\left(P S_{\mathbb{C}}, s_{\alpha} \times \operatorname{sgn} e^{\rho}\right)=M\left(I\left(H, \operatorname{sgn} e^{\rho}\right), s_{\alpha} \cdot P S_{\mathbb{C}}\right) \\
& =M\left(P S_{\mathrm{sgn}}, P S_{\mathrm{sgn}}\right)=1 \\
a\left(P S_{\mathbb{C}}, \operatorname{sgn} e^{-\rho}\right) & =M\left(I\left(H, s_{\alpha} \times \operatorname{sgn} e^{\rho}\right), P S_{\mathbb{C}}\right)=M\left(P S_{\mathrm{sgn}}, P S_{\mathrm{sgn}}\right)=1
\end{aligned}
$$


The formulas for the characters of the principal series are given in Example 8.15 .

Next consider the elementary character formula for the discrete series representation on $H=S^{1}$, with $q_{H}=1, B\left(\Delta^{+}\right)=0$, and $I\left(H, e^{\rho}\right)=D S$ :

$$
\begin{aligned}
a\left(D S, e^{\rho}\right) & =-M(D S, D S)=-1 \\
a\left(D S, e^{-\rho}\right) & =-M\left(D S, s_{\alpha} \cdot D S\right)=-M\left(D S, P S_{\mathbb{C}}+P S_{\mathrm{sgn}}-D S\right)=1 .
\end{aligned}
$$

So:

$$
\theta_{D S}\left(e^{i \theta}\right)=\frac{-e^{i \theta}+e^{i \theta}}{e^{i \theta}-e^{-i \theta}}=-1
$$

Note that $\theta_{D S}\left(e^{i \theta}\right)=-\theta_{\mathbb{C}}\left(e^{i \theta}\right)$, which follows from (14.8)(a).

The most interesting case is the discrete series on $H=\mathbb{R}^{*}$, with $q_{H}=$ $B\left(\Delta^{+}\right)=0, I\left(\mathbb{R}^{*}, e^{\rho}\right)=P S_{\mathbb{C}}, I\left(\mathbb{R}^{*}, \operatorname{sgn} e^{\rho}\right)=P S_{-}:$

$$
\begin{aligned}
a\left(D S, e^{\rho}\right) & =M\left(I\left(\mathbb{R}^{*}, e^{\rho}\right), D S\right)=M\left(P S_{\mathbb{C}}, D S\right)=0 \\
a\left(D S, \operatorname{sgn} e^{\rho}\right) & =M\left(I\left(\mathbb{R}^{*}, \operatorname{sgn} e^{\rho}\right), D S\right)=M\left(P S_{\mathrm{sgn}}, D S\right)=0 \\
a\left(D S, e^{-\rho}\right) & =a\left(D S, s_{\alpha} \times e^{\rho}\right)=M\left(I\left(\mathbb{R}^{*}, e^{\rho}\right), s_{\alpha} \cdot D S\right) \\
& =M\left(P S_{\mathbb{C}}, P S_{\mathbb{C}}+P S_{\mathrm{sgn}}-D S\right)=1 \\
a\left(D S, \operatorname{sgn} e^{-\rho}\right) & =a\left(D S, s_{\alpha} \operatorname{sgn} e^{-\rho}\right)=M\left(I\left(\mathbb{R}^{*}, \operatorname{sgn} e^{\rho}\right), s_{\alpha} \cdot D S\right) \\
& =M\left(P S_{\mathrm{sgn}}, P S_{\mathbb{C}}+P S_{\mathrm{sgn}}-D S\right)=1
\end{aligned}
$$

Therefore for $|x|>1$ we have:

$$
\begin{aligned}
\theta_{D S}(x) & =\frac{z^{-1}+\operatorname{sgn}(x) z^{-1}}{\left(1-\frac{1}{x}\right) z} \quad\left(\text { where } z^{2}=x\right) \\
& =\frac{|x|^{-\frac{1}{2}}+\operatorname{sgn}(x)|x|^{-\frac{1}{2}}}{|x|^{\frac{1}{2}}-|x|^{-\frac{1}{2}}} \\
& = \begin{cases}\frac{2 x^{-\frac{1}{2}}}{|x|^{\frac{1}{2}}-|x|^{-\frac{1}{2}}} & x>1 \\
0 & x<-1 .\end{cases}
\end{aligned}
$$

Another way to write this is

$$
\theta_{D S}(x)=\frac{1+\operatorname{sgn}(x)}{x-1} \quad(|x|>1) .
$$


Also note that

$$
\theta_{D S}(x)=\frac{-|x|^{\frac{1}{2}}-\operatorname{sgn}(x)|x|^{\frac{1}{2}}}{|x|^{\frac{1}{2}}-|x|^{-\frac{1}{2}}} \quad(|x|<1) .
$$

\subsection{Discrete series representations of $S p(4, \mathbb{R})$}

We now calculate the characters of the discrete series representations of $S p(4, \mathbb{R})$. We make some use of the atlas software; see [2] for an introduction. The formulas we obtain can be readily shown to agree with those of $[13,6.2]$, and (with a little more work) with [15, page 499] and [12, (4.9)].

There are 4 discrete series representations of $S p(4, \mathbb{R})$ with infinitesimal character $\rho$. Two of these are holomorphic/anti-holomorphic (in chambers with a compact simple root), and the other two are large (in a chamber in which both simple roots are noncompact). The only representations we need to consider are in the block of these representations, since this is preserved by the coherent continuation action. This block is the span in the Grothendieck group of 12 standard representations, or the corresponding irreducible representations. These can be read off from the output of the block command in atlas, and are numbered 0 to 11 . Representations $0-3$ are in the discrete series; 0,1 are large, and 2,3 are holomorphic/anti-holomorphic. For more information on blocks, and the atlas software see [2, Example 10.3].

Here is the output of the block command:

\begin{tabular}{|c|c|c|c|c|c|c|c|c|}
\hline $0(0,6):$ & 0 & {$[i 1, i 1]$} & 1 & 2 & $(4, *)$ & $(5, *)$ & 0 & e \\
\hline $1(1,6):$ & 0 & {$[i 1, i 1]$} & 0 & 3 & $(4, *)$ & $(6, *)$ & 0 & e \\
\hline $2(2,6):$ & 0 & {$[i c, i 1]$} & 2 & 0 & $(*, *)$ & $(5, *)$ & 0 & $\mathrm{e}$ \\
\hline $3(3,6):$ & 0 & {$[i c, i 1]$} & 3 & 1 & $(*, *)$ & $(6, *)$ & 0 & e \\
\hline $4(4,5):$ & 1 & {$[\mathrm{r} 1, \mathrm{C}+]$} & 4 & 9 & $(0,1)$ & $(*, *)$ & 1 & 1 \\
\hline $5(5,4):$ & 1 & {$[\mathrm{C}+, \mathrm{r} 1]$} & 7 & 5 & $(*, *)$ & $(0,2)$ & 2 & 2 \\
\hline $6(6,4):$ & 1 & {$[\mathrm{C}+, \mathrm{r} 1]$} & 8 & 6 & $(*, *)$ & $(1,3)$ & 2 & 2 \\
\hline $7(7,3):$ & 2 & {$[\mathrm{C}-, \mathrm{i} 1]$} & 5 & 8 & $(*, *)$ & $(10, *)$ & 2 & $1,2,1$ \\
\hline $8(8,3):$ & 2 & {$[\mathrm{C}-, \mathrm{i} 1]$} & 6 & 7 & $(*, *)$ & $(10, *)$ & 2 & $1,2,1$ \\
\hline $9(9,2):$ & 2 & {$[\mathrm{i} 2, \mathrm{C}-]$} & 9 & 4 & $(10,11)$ & $(*, *)$ & 1 & $2,1,2$ \\
\hline $10(10,0)$ & 3 & {$[r 2, r 1]$} & 11 & 10 & $(9, *)$ & $(7,8)$ & 3 & $2,1,2,1$ \\
\hline $11(10,1)$ & 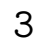 & {$[\mathrm{r} 2, \mathrm{rn}]$} & 10 & 11 & $(9, *)$ & $(*, *)$ & 3 & $2,1,2,1$ \\
\hline
\end{tabular}


This block also has 2 principal series representations, numbers 10 and 11 . Standard representation 10 is the spherical principal series. Its irreducible quotient $\pi(10)$ is the trivial representation. Standard module 11 is the unique nonspherical principal series representation with trivial infinitesimal and central characters. This is reducible, and $\pi(11)$ is infinite dimensional.

(There are 6 other irreducible representations of $S p(4, \mathbb{R})$ with infinitesimal character $\rho$. These are not in the previous block, have nontrivial central character, and include two minimal principle series representations, one of which is irreducible.)

\subsubsection{Split Cartan subgroup}

Let $H(\mathbb{R})$ be a split Cartan subgroup. Identify $H(\mathbb{R})$ with $\mathbb{R}^{* 2}$ such that the roots are $(x, y) \rightarrow x^{ \pm 2}, y^{ \pm 2}, x^{ \pm 1} y^{ \pm 1}$. Write $(a, b)$ for the character $(x, y) \rightarrow$ $x^{a} y^{b}$. Choose simple roots $\alpha=(1,-1)$ and $\beta=(0,2)$, which define $\Delta^{+}$, and give $\rho=(2,1)$. Write $D$ for the Weyl denominator defined by $\Delta^{+}$. Since $\rho$ exponentiates we may ignore the cover. Finally let $\chi(x, y)=\operatorname{sgn}(x y)$.

In the setting of Corollary (12.5) and the subsequent discussion we only need to consider $\Lambda=e^{\rho}$ or $\chi e^{\rho}$. In this terminology

$$
\begin{aligned}
I\left(H, e^{\rho}\right) & =I(10) \\
I\left(H, \chi e^{\rho}\right) & =I(11) .
\end{aligned}
$$

First we take $\pi$ to be the large discrete series representation $\pi(0)=I(0)$. With a little care we may ignore the difference between $W_{a}$ and $W$. Using (14.12) and (12.5) we have

$$
\begin{aligned}
a\left(\pi, e^{w^{-1} \rho}\right) & =M(I(10), w \cdot \pi) \\
a\left(\pi, \chi e^{w^{-1} \rho}\right) & =M(I(11), w \cdot \pi)
\end{aligned}
$$

The first column of the next table gives $w \in W$ as a product of simple reflections $s_{\alpha}, s_{\beta}$, which we label 1,2 , respectively. The second column gives $w^{-1} \rho$. There is a subtlety here: because of (2.2), if you view the first column as an element $w^{\prime}$ of $W$ (not $W_{a}$ ), then the second column is $w^{\prime} \rho\left(\right.$ not $w^{-1} \rho$ ).

We compute $w \cdot \pi$, as a sum of standard modules, using the output of the block command. The application coherentContinuation, available at www.liegroups.org/software/helpers is very useful for expediting this calculation. We omit the details how to do this, but the result is listed in 
column 3 of the next table. For example the second row indicates $s_{\alpha} \cdot \pi=$ $-I(1)+I(4)$ (the coefficients are all \pm 1 ).

By $(14.13) a\left(\pi, e^{w^{-1} \rho}\right)$ (respectively $\left.a\left(\pi, \chi e^{w^{-1} \rho}\right)\right)$ is computed as the multiplicity of $I(10)$ (respectively $I(11)$ ) in column 3 , which is given in columns 4 and 5 .

\begin{tabular}{|c|c|c|c|c|}
\hline$w$ & $w^{-1} \rho$ & $w \cdot \pi$ & $a\left(\pi, e^{w^{-1} \rho}\right)$ & $a\left(\pi, \chi e^{w^{-1} \rho}\right)$ \\
\hline $\mathrm{e}$ & $(2,1)$ & 0 & 0 & 0 \\
\hline 1 & $(1,2)$ & $-1+4$ & 0 & 0 \\
\hline 2 & $(2,-1)$ & $-2+5$ & 0 & 0 \\
\hline 12 & $(-1,2)$ & $2+7$ & 0 & 0 \\
\hline 21 & $(1,-2)$ & $3-6+9$ & 0 & 0 \\
\hline 121 & $(-2,1)$ & $-3-8-9+10+11$ & 1 & 1 \\
\hline 212 & $(-1,-2)$ & $-0+5-8+10$ & 1 & 0 \\
\hline 1212 & $(-2,-1)$ & $1-4-6+7+11$ & 0 & 1 \\
\hline
\end{tabular}

Let

$$
H(\mathbb{R})_{+}=\{(x, y)|| x|>| y \mid>1\} .
$$

From the table we conclude that on $H(\mathbb{R})_{+}$we have

$$
\left.\left(D \theta_{\pi}\right)(x, y)\right)=x^{-2} y+\operatorname{sgn}(x y) x^{-2} y+x^{-1} y^{-2}+\operatorname{sgn}(x y) x^{-2} y^{-1}
$$

Perhaps a more familiar way to write this is as follows. Let $t=\left(\epsilon_{1}, \epsilon_{2}\right)$ $\left(\epsilon_{i}= \pm 1\right)$. Write $(a, b)$ for the differential of the character $(a, b)$ of $H$. Suppose $X \in \mathfrak{h}_{0}$, and assume $e^{X} \in H(\mathbb{R})_{+}$. Let $H(\mathbb{R})^{0}$ identity component of $H(\mathbb{R})$. Then the character formula on $H(\mathbb{R})^{0} \cap H(\mathbb{R})_{+}$is

$$
\left(D \theta_{\pi}\right)(\exp (X))=2 e^{(-2,1) X}+e^{(-1,-2) X}+e^{(-2,-1) X} .
$$

It is worth pointing out that all nonzero KLV polynomials are 1, in spite of the 2 appearing in this formula (but this is explained by the fact that $1+1=2)$. We also see, as is evident from the central character and the fact that $e^{\rho}(-1,-1)=-1$, that

$$
\left(D \theta_{\pi}\right)(-\exp (X))=-\left(D \theta_{\pi}\right)(\exp X)
$$

On the other hand if $t=(\epsilon,-\epsilon)(\epsilon= \pm 1)$ then on $t H(\mathbb{R})^{0} \cap H(\mathbb{R})_{+}$we have:

$$
\left(D \theta_{\pi}\right)(t \exp (X))=\epsilon\left(e^{(-1,-2) X}+e^{(-2,-1) X}\right) .
$$


Formula (14.16) has the advantage that it holds on all of $H(\mathbb{R})_{+}$.

More succinctly, here are character formulas for the other discrete series representations $\pi(1)$ (large) and $\pi(2), \pi(3)$ (holomorphic/antiholomorphic). In the column $w \cdot \pi(i)$ we only list the terms 10,11 which occur.

In this table the entry in column $6,7,8$ in the multiplicity of $I(10)$ in column $3,4,5$, respectively. The entry in column $9,10,11$ is the multiplicity of $I(11)$ in column $3,4,5$, respectively.

\begin{tabular}{|c|c|c|c|c|c|c|c|c|c|c|}
\hline & & \multicolumn{3}{|c|}{$w \cdot \pi(i)$} & \multicolumn{3}{|c|}{$a\left(\pi(i), e^{w^{-1} \rho}\right)$} & \multicolumn{3}{|c|}{$a\left(\pi(i), \chi e^{w^{-1} \rho}\right)$} \\
\hline$w$ & $w^{-1} \rho$ & $\pi(1)$ & $\pi(2)$ & $\pi(3)$ & $\pi(1)$ & $\pi(2)$ & $\pi(3)$ & $\overline{\pi(1)}$ & $\pi(2)$ & $\pi(3)$ \\
\hline 121 & $(-2,1)$ & $10+11$ & $*$ & * & 1 & 0 & 0 & 1 & 0 & 0 \\
\hline 212 & $(-1,-2)$ & 10 & 10 & 10 & 1 & 1 & 1 & 0 & 0 & 0 \\
\hline 1212 & $(-2,-1)$ & 11 & -10 & -10 & 0 & -1 & -1 & 1 & 0 & 0 \\
\hline
\end{tabular}

We conclude that the characters of $\pi(0)$ and $\pi(1)$ are equal on $H(\mathbb{R})$. Moreover ( still on $H(\mathbb{R})_{+}$) we have

$$
\left(D \theta_{\pi(k)}\right)(x, y)=x^{-1} y^{-2}-x^{-2} y^{-1} \quad(k=2,3) .
$$

Alternatively, with $t=\left(\epsilon_{1}, \epsilon_{2}\right)$ :

$$
\left(D \theta_{\pi(k)}(t \exp (X))=\epsilon_{1} e^{(-1,-2) X}-\epsilon_{2} e^{(-2,-1) X}\right.
$$

\subsubsection{Cartan \#2: $H(\mathbb{R}) \simeq \mathbb{R}^{*} \times S^{1}$}

We briefly consider a Cartan subgroup with $H(\mathbb{R}) \simeq \mathbb{R}^{*} \times S^{1}$; this is Cartan $\# 2$ in atlas. Identify $H(\mathbb{R})$ with $\mathbb{R}^{*} \times S^{1}$, and choose simple roots $\alpha, \beta$ so that $\alpha\left(x, e^{i \theta}\right)=x e^{-i \theta}, \quad \beta\left(x, e^{i \theta}\right)=e^{2 i \theta}$. Write $(a, b)$ for the character $\left(x, e^{i \theta}\right) \rightarrow x^{a} e^{i b \theta}$, so $e^{\rho}=(2,1)$.

In this case $q_{H}=1, B\left(\Delta^{+}\right)=0$, and $\epsilon\left(\Delta_{i}^{+}, e^{\rho}\right)=1$. We can check that $I\left(H, e^{\rho}\right)=I(8)$, and conclude

$$
a\left(\pi(i), e^{w^{-1} \rho}\right)=-M(I(8), w \cdot \pi(i)) .
$$

Using atlas we compute the following table.

\begin{tabular}{|c|c|c|c|c|c|c|c|c|c|}
\hline \multicolumn{2}{|c|}{} & \multicolumn{4}{|c|}{$w \cdot \pi(i)$} & \multicolumn{4}{c|}{$a\left(\pi(i), e^{w^{-1}} \rho\right.$} \\
\hline$w$ & $w^{-1} \rho$ & $\pi(0)$ & $\pi(1)$ & $\pi(2)$ & $\pi(3)$ & $\pi(0)$ & $\pi(1)$ & $\pi(2)$ & $\pi(3)$ \\
\hline 12 & $(-1,2)$ & $*$ & 8 & $*$ & 8 & 0 & -1 & 0 & -1 \\
\hline 121 & $(-2,1)$ & -8 & $*$ & $*$ & -8 & 1 & 0 & 0 & 1 \\
\hline 212 & $(-1,-2)$ & -8 & $*$ & -8 & $*$ & 1 & 0 & 1 & 0 \\
\hline 1212 & $(-2,-1)$ & $*$ & 8 & 8 & $*$ & 0 & -1 & -1 & 0 \\
\hline
\end{tabular}


Therefore on

$$
H(\mathbb{R})_{+}=\left\{\left(x, e^{i \theta}\right)|| x \mid>1\right\}
$$

we have formulas
$(14.20)(\mathrm{a})$
$\left(D \theta_{\pi(0)}\right)\left(x e^{i \theta}\right)=x^{-1} e^{-2 i \theta}+x^{-2} e^{i \theta}$
$(14.20)(\mathrm{b})$
$\left(D \theta_{\pi(1)}\right)\left(x e^{i \theta}\right)=-x^{-1} e^{2 i \theta}-x^{-2} e^{-i \theta}$
$(14.20)(\mathrm{c})$
$\left(D \theta_{\pi(2)}\right)\left(x e^{i \theta}\right)=x^{-1} e^{-2 i \theta}-x^{-2} e^{-i \theta}$
$(14.20)(d)$
$\left(D \theta_{\pi(3)}\right)\left(x e^{i \theta}\right)=-x^{-1} e^{2 i \theta}+x^{-2} e^{i \theta}$.

14.3.3 Cartan \#1: $H(\mathbb{R}) \simeq \mathbb{C}^{*}$

Finally suppose $H(\mathbb{R}) \simeq \mathbb{C}^{*}$ (Cartan \#3 in atlas). Choose the isomorphism, and simple roots $\alpha, \beta$, so that

$$
\alpha(z)=z / \bar{z}, \quad \beta(z)=\bar{z}^{2} .
$$

Write $(a, b)$ for the character $z \rightarrow z^{a} \bar{z}^{b}$, i.e. $e^{x+i y} \rightarrow e^{(a+b) x+(a-b) i y}$. In particular $e^{\rho}=(2,1)$, and $e^{\rho}\left(e^{x+i y}\right)=e^{3 x+i y}$.

In this case $I\left(H, e^{\rho}\right)=I(9), q_{H}=1, \epsilon\left(\Delta_{i}^{+}, e^{\rho}\right)=1, B\left(\Delta^{+}\right)=0$, and

$$
a\left(\pi(i), e^{w^{-1} \rho}\right)=-M(I(9), w \cdot \pi(i)) .
$$

Using atlas we compute

\begin{tabular}{|c|c|c|c|c|c|c|c|c|c|}
\hline \multicolumn{2}{|c|}{} & \multicolumn{4}{|c|}{$w \cdot \pi(i)$} & \multicolumn{4}{c|}{$a\left(\pi(i), e^{w^{-1}} \rho\right.$} \\
\hline$w$ & $w^{-1} \rho$ & $\pi(0)$ & $\pi(1)$ & $\pi(2)$ & $\pi(3)$ & $\pi(0)$ & $\pi(1)$ & $\pi(2)$ & $\pi(3)$ \\
\hline 21 & $(1,-2)$ & 9 & 9 & $*$ & $*$ & 1 & 1 & 0 & 0 \\
\hline 121 & $(-2,1)$ & -9 & -9 & $*$ & $*$ & -1 & -1 & 0 & 0 \\
\hline 212 & $(-1,-2)$ & $*$ & $*$ & -9 & -9 & 0 & 0 & -1 & -1 \\
\hline 1212 & $(-2,-1)$ & $*$ & $*$ & 9 & 9 & 0 & 0 & 1 & 1 \\
\hline
\end{tabular}

Therefore on

$$
H(\mathbb{R})_{+}=\left\{e^{x+i y} \mid x>0\right\}
$$

we have

$$
\left(D \theta_{\pi(i)}\right)\left(e^{x+i y}\right)= \begin{cases}-e^{-x+3 i y}+e^{-x-3 i y} & i=0,1 \\ e^{-3 x+i y}-e^{-3 x-i y} & i=1,2\end{cases}
$$




\subsection{Character Table for $S p(4, \mathbb{R})$ at $\rho$}

Without giving any more details of the calculations, here is complete information about the characters of the irreducible representations of $S p(4, \mathbb{R})$ in the block of the trivial representation.

We use notation for the four Cartan subgroups $S^{1} \times S^{1}, \mathbb{C}^{*}, \mathbb{R}^{*} \times \S^{1}, \mathbb{R}^{*} \times \mathbb{R}^{*}$ as in Section 14.3.1, 14.3.2 and 14.3.3. (We haven't considered the compact Cartan subgroup yet; the notation is obvious here, and we choose $\Delta^{+}$so that $\rho=(2,1)$ as usual.) For each Cartan subgroup we have fixed a choice of $\Delta^{+}$, and $D$ is the corresponding Weyl denominator. The characters $\Lambda$ of $H(\mathbb{R})$ with $d \Lambda \in W \rho$ are parametrized in each case by $\{( \pm a, \pm b)\}$ with $(a, b)=(2,1)$ or $(1,2)$. In the case of the split Cartan subgroup there are two characters $(a, b)$ and $\chi(a, b)$ (see Section 14.3.1).

As discussed in Section 14.3 the block of the trivial representation consists of 12 standard modules $I(i)$, and their corresponding irreducible representations $\pi(i)$, with $0 \leq i \leq 11$. The character formulas for the irreducible representations, in terms of standard modules are as follows. This was computed using the klbasis command; and may also be found in [23].

$$
\begin{aligned}
\pi(0) & =I(0) \\
\pi(1) & =I(1) \\
\pi(2) & =I(2) \\
\pi(3) & =I(3) \\
\pi(4) & =-I(0)-I(1)+I(4) \\
\pi(5) & =-I(0)-I(2)+I(5) \\
\pi(6) & =-I(1)-I(3)+I(6) \\
\pi(7) & =I(0)+I(1)+I(2)-I(4)-I(5))+I(7) \\
\pi(8) & =I(0)+I(1)+I(3)-I(4)-I(6)+I(8) \\
\pi(9) & =I(0)+I(1)+I(2)+I(3)-I(4)-I(5)-I(6)+I(9) \\
\pi(10) & =-I(0)-I(1)-I(2)-I(3)+I(4)+I(5)+I(6)-I(7)-I(8)-I(9)+I(10) \\
\pi(11) & =-I(2)-I(3)-I(9)+I(11)
\end{aligned}
$$

Each row in the following tables gives the character formula for a single standard or irreducible module, on the given Cartan subgroup. 
For example the first row in the first table below says the formula for the large discrete series representation $I(0)=\pi(0)$ on the compact Cartan subgroup is:

$$
\left(D \theta_{I(0)}\right)\left(e^{i \theta_{1}}, e^{i \theta_{2}}\right)=-e^{2 i \theta_{1}-i \theta_{2}}+e^{-i \theta_{1}+2 i \theta_{2}}
$$

The last entry in the second table gives

$$
\left(D \theta_{\pi(11)}\right)\left(e^{i \theta_{1}}, e^{i \theta_{2}}\right)=-e^{2 i \theta_{1}+i \theta_{2}}+e^{i \theta_{1}+2 i \theta_{2}}+e^{-i \theta_{1}-2 i \theta_{2}}-e^{-2 i \theta_{1}-i \theta_{2}}
$$

\section{Compact Cartan subgroup}

$$
\begin{aligned}
(a, b) & :\left(e^{i \theta_{1}}, e^{i \theta_{2}}\right) \rightarrow e^{a i \theta_{1}+b i \theta_{2}} \\
H(\mathbb{R})_{+} & =H(\mathbb{R})
\end{aligned}
$$

\begin{tabular}{|c|c|c|c|c|c|c|c|c|}
\hline & \multicolumn{7}{|c|}{$S^{1} \times S^{1}:$ Standard Modules } \\
\hline$I$ & $(2,1)$ & $(1,2)$ & $(2,-1)$ & $(-1,2)$ & $(1,-2)$ & $(-2,1)$ & $(-1,-2)$ & $(-2,-1)$ \\
\hline$I(0)$ & & & -1 & 1 & & & & \\
\hline$I(1)$ & & & & & 1 & -1 & & \\
\hline$I(2)$ & 1 & -1 & & & & & & \\
\hline$I(3)$ & & & & & & & -1 & 1 \\
\hline
\end{tabular}

\begin{tabular}{|c|c|c|c|c|c|c|c|c|}
\hline & \multicolumn{7}{|c|}{$S^{1} \times S^{1}$ : Irreducible Modules } \\
\hline$\pi$ & $(2,1)$ & $(1,2)$ & $(2,-1)$ & $(-1,2)$ & $(1,-2)$ & $(-2,1)$ & $(-1,-2)$ & $(-2,-1)$ \\
\hline$\pi(0)$ & & & -1 & 1 & & & & \\
\hline$\pi(1)$ & & & & & 1 & -1 & & \\
\hline$\pi(2)$ & 1 & -1 & & & & & & \\
\hline$\pi(3)$ & & & & & & & -1 & 1 \\
\hline$\pi(4)$ & & & 1 & -1 & -1 & 1 & & \\
\hline$\pi(5)$ & -1 & 1 & 1 & -1 & & & & \\
\hline$\pi(6)$ & & & & & -1 & 1 & 1 & -1 \\
\hline$\pi(7)$ & 1 & -1 & -1 & 1 & 1 & -1 & & \\
\hline$\pi(8)$ & & & -1 & 1 & 1 & -1 & -1 & 1 \\
\hline$\pi(9)$ & 1 & -1 & -1 & 1 & 1 & -1 & -1 & 1 \\
\hline$\pi(10)$ & -1 & 1 & 1 & -1 & -1 & 1 & 1 & -1 \\
\hline$\pi(11)$ & -1 & 1 & & & & & 1 & -1 \\
\hline
\end{tabular}

The identity (6.6)(b) is clear in the table, for $w$ the short simple reflection, which is $W(G(\mathbb{R}), H(\mathbb{R})) \subset W_{i}=W$. 
Cartan \#2: $\mathbb{R}^{*} \times S^{1}$

$$
\begin{gathered}
(a, b):\left(x, e^{i \theta}\right) \rightarrow x^{2} e^{b i \theta} \\
H(\mathbb{R})_{+}=\left\{\left(x, e^{i \theta}\right)|| x \mid>1\right\}
\end{gathered}
$$

See Section 14.3.2.

\begin{tabular}{|c|c|c|c|c|c|c|c|c|}
\hline & \multicolumn{7}{|c|}{$\mathbb{R}^{*} \times S^{1}:$ Standard Modules } \\
\hline$I$ & $(2,1)$ & $(1,2)$ & $(2,-1)$ & $(-1,2)$ & $(1,-2)$ & $(-2,1)$ & $(-1,-2)$ & $(-2,-1)$ \\
\hline$I(0)$ & & & & & & 1 & 1 & \\
\hline$I(1)$ & & & & -1 & & & & -1 \\
\hline$I(2)$ & & & & & & & 1 & -1 \\
\hline$I(3)$ & & & & -1 & & 1 & & \\
\hline$I(5)$ & & & & & 1 & & 1 & \\
\hline$I(6)$ & & -1 & & -1 & & & & \\
\hline$I(7)$ & & & 1 & & & & & 1 \\
\hline$I(8)$ & -1 & & & & & -1 & & \\
\hline
\end{tabular}

\begin{tabular}{|c|c|c|c|c|c|c|c|c|}
\hline & \multicolumn{7}{|c|}{$\mathbb{R}^{*} \times S^{1}:$ Irreducible Modules } \\
\hline$\pi$ & $(2,1)$ & $(1,2)$ & $(2,-1)$ & $(-1,2)$ & $(1,-2)$ & $(-2,1)$ & $(-1,-2)$ & $(-2,-1)$ \\
\hline$\pi(0)$ & & & & & & 1 & 1 & \\
\hline$\pi(1)$ & & & & -1 & & & & -1 \\
\hline$\pi(2)$ & & & & & & & 1 & -1 \\
\hline$\pi(3)$ & & & & -1 & & 1 & & \\
\hline$\pi(4)$ & & & & 1 & & -1 & -1 & 1 \\
\hline$\pi(5)$ & & & & & 1 & -1 & -1 & 1 \\
\hline$\pi(6)$ & & -1 & & 1 & & -1 & & 1 \\
\hline$\pi(7)$ & & & 1 & -1 & -1 & 1 & 1 & -1 \\
\hline$\pi(8)$ & -1 & 1 & & -1 & & 1 & 1 & -1 \\
\hline$\pi(9)$ & & 1 & & -1 & -1 & 2 & 1 & -2 \\
\hline$\pi(10)$ & 1 & -1 & -1 & 1 & 1 & -1 & -1 & 1 \\
\hline$\pi(11)$ & & & & 1 & 0 & -1 & -1 & 1 \\
\hline
\end{tabular}

The first four lines of the tables are equivalent to (14.20)(a-d). 


\section{Cartan \#1: $\mathbb{C}^{*}$}

$$
\begin{aligned}
(a, b) & : e^{x+i y} \rightarrow e^{(a+b) x+(a-b) i y} \\
H(\mathbb{R})_{+} & =\left\{\left(e^{x+i y} \mid x>0\right\}\right.
\end{aligned}
$$

See Section 14.3.3.

\begin{tabular}{|c|c|c|c|c|c|c|c|c|}
\hline & \multicolumn{7}{|c|}{$\mathbb{C}^{*}:$ Standard Modules } \\
\hline$I$ & $(2,1)$ & $(1,2)$ & $(2,-1)$ & $(-1,2)$ & $(1,-2)$ & $(-2,1)$ & $(-1,-2)$ & $(-2,-1)$ \\
\hline$I(0)$ & & & & & -1 & 1 & & \\
\hline$I(1)$ & & & & & -1 & 1 & & \\
\hline$I(2)$ & & & & & & & 1 & -1 \\
\hline$I(3)$ & & & & & & & 1 & -1 \\
\hline$I(4)$ & & & -1 & 1 & -1 & 1 & & \\
\hline$I(9)$ & -1 & 1 & & & & & -1 & 1 \\
\hline
\end{tabular}

\begin{tabular}{|c|c|c|c|c|c|c|c|c|}
\hline & \multicolumn{7}{|c|}{$\mathbb{C}^{*}$ : Irreducible Modules } \\
\hline$\pi$ & $(2,1)$ & $(1,2)$ & $(2,-1)$ & $(-1,2)$ & $(1,-2)$ & $(-2,1)$ & $(-1,-2)$ & $(-2,-1)$ \\
\hline$\pi(0)$ & & & & & -1 & 1 & & \\
\hline$\pi(1)$ & & & & & -1 & 1 & & \\
\hline$\pi(2)$ & & & & & & & 1 & -1 \\
\hline$\pi(3)$ & & & & & & & 1 & -1 \\
\hline$\pi(4)$ & & & -1 & 1 & 1 & -1 & & \\
\hline$\pi(5)$ & & & & & 1 & -1 & -1 & 1 \\
\hline$\pi(6)$ & & & & & 1 & -1 & -1 & 1 \\
\hline$\pi(7)$ & & & 1 & -1 & -1 & 1 & 1 & -1 \\
\hline$\pi(8)$ & & & 1 & -1 & -1 & 1 & 1 & -1 \\
\hline$\pi(9)$ & -1 & 1 & 1 & -1 & -1 & 1 & 1 & -1 \\
\hline$\pi(10)$ & 1 & -1 & -1 & 1 & 1 & -1 & -1 & 1 \\
\hline$\pi(11)$ & 1 & -1 & & & & & -1 & 1 \\
\hline
\end{tabular}

The first four lines of the tables are equivalent to (14.22).

As in the case of the compact Cartan subgroup the identity (6.6)(b) is clear; the short simple reflection is in $W(G(\mathbb{R}), H(\mathbb{R})) \cap W_{i}$.

\section{Split Cartan subgroup}

In the next two tables the two entries in an ordered pair in a column labelled $(a, b)$ gives the multipicity of the characters

$$
(a, b):(x, y) \rightarrow x^{a} y^{b}, \quad(x, y) \rightarrow \operatorname{sgn}(x y) x^{a} y^{b}
$$


respectively. For example the last entry in the row for $\pi(4)$ is $(1,-1)$ in a column labelled $(-2,-1)$; this means the function $(x, y) \rightarrow x^{-2} y^{-1}-$ $\operatorname{sgn}(x y) x^{-2} y^{-1}$. Also

$$
H(\mathbb{R})_{+}=\{(x, y)|| x|>| y \mid>0\} .
$$

See Section 14.3.3.

\begin{tabular}{|c|c|c|c|c|c|c|c|c|}
\hline & \multicolumn{7}{|c|}{$\mathbb{R}^{*} \times \mathbb{R}^{*}:$ Standard Modules } \\
\hline$I$ & $(2,1)$ & $(1,2)$ & $(2,-1)$ & $(-1,2)$ & $(1,-2)$ & $(-2,1)$ & $(-1,-2)$ & $(-2,-1)$ \\
\hline$I(0)$ & & & & & & 1,1 & 1,0 & 0,1 \\
\hline$I(1)$ & & & & & & 1,1 & 1,0 & 0,1 \\
\hline$I(2)$ & & & & & & & 1,0 & $-1,0$ \\
\hline$I(3)$ & & & & & & & 1,0 & $-1,0$ \\
\hline$I(4)$ & & & & 1,1 & & 1,1 & 1,1 & 1,1 \\
\hline$I(5)$ & & & & & 1,0 & 0,1 & 1,0 & 0,1 \\
\hline$I(6)$ & & & & & 1,0 & 0,1 & 1,0 & 0,1 \\
\hline$I(7)$ & & & 1,0 & 0,1 & & & 0,1 & 1,0 \\
\hline$I(8)$ & & & 1,0 & 0,1 & & & 0,1 & 1,0 \\
\hline$I(9)$ & & 1,1 & & & 1,1 & 1,1 & & 1,1 \\
\hline$I(10)$ & 1,0 & 0,1 & 1,0 & 0,1 & 0,1 & 1,0 & 0,1 & 1,0 \\
\hline$I(11)$ & 0,1 & 1,0 & 0,1 & 1,0 & 1,0 & 0,1 & 1,0 & 0,1 \\
\hline
\end{tabular}

\begin{tabular}{|c|c|c|c|c|c|c|c|c|}
\hline & \multicolumn{7}{|c|}{$\mathbb{R}^{*} \times \mathbb{R}^{*}:$ Irreducible Modules } \\
\hline$\pi$ & $(2,1)$ & $(1,2)$ & $(2,-1)$ & $(-1,2)$ & $(1,-2)$ & $(-2,1)$ & $(-1,-2)$ & $(-2,-1)$ \\
\hline$\pi(0)$ & & & & & & 1,1 & 1,0 & 0,1 \\
\hline$\pi(1)$ & & & & & & 1,1 & 1,0 & 0,1 \\
\hline$\pi(2)$ & & & & & & & 1,0 & $-1,0$ \\
\hline$\pi(3)$ & & & & & & & 1,0 & $-1,0$ \\
\hline$\pi(4)$ & & & & 1,1 & & $-1,-1$ & $-1,1$ & $1,-1$ \\
\hline$\pi(5)$ & & & & & 1,0 & $-1,0$ & $-1,0$ & 1,0 \\
\hline$\pi(6)$ & & & & & 1,0 & $-1,0$ & $-1,0$ & 1,0 \\
\hline$\pi(7)$ & & & 1,0 & $-1,0$ & $-1,0$ & 1,0 & 1,0 & $-1,0$ \\
\hline$\pi(8)$ & & & 1,0 & $-1,0$ & $-1,0$ & 1,0 & 1,0 & $-1,0$ \\
\hline$\pi(9)$ & & 1,1 & & $-1,-1$ & $-1,1$ & 2,0 & $1,-1$ & $-2,0$ \\
\hline$\pi(10)$ & 1,0 & $-1,0$ & $-1,0$ & 1,0 & 1,0 & $-1,0$ & $-1,0$ & 1,0 \\
\hline$\pi(11)$ & 0,1 & $0,-1$ & 0,1 & 1,0 & $0,-1$ & $-1,0$ & $-1,0$ & 1,0 \\
\hline
\end{tabular}

The first four lines of the tables are equivalent to (14.16) and (14.18). 


\section{References}

[1] J. Adams, M. van Leeuwen, A. Paul, P. Trapa, D. A. Jr. Vogan, and W.-L. Yee. Unitary dual of real reductive groups. preprint.

[2] Jeffrey Adams. Guide to the atlas software: Computational representation theory of real reductive groups. In Representation Theory of Real Reductive Groups, Proceedings of Conference at Snowbird, July 2006, Contemp. Math. Amer. Math. Soc., 2008.

[3] Jeffrey Adams, Dan Barbasch, and David A. Vogan, Jr. The Langlands classification and irreducible characters for real reductive groups, volume 104 of Progress in Mathematics. Birkhäuser Boston Inc., Boston, MA, 1992.

[4] Jeffrey Adams and David A. Vogan, Jr. L-groups, projective representations, and the Langlands classification. Amer. J. Math., 114(1):45-138, 1992.

[5] Juan Bigeon and Jorge Vargas. A new formula for discrete series characters on split groups. J. Lie Theory, 16(2):329-349, 2006.

[6] M. Goresky, R. Kottwitz, and R. MacPherson. Discrete series characters and the Lefschetz formula for Hecke operators. Duke Math. J., 89(3):477-554, 1997.

[7] Harish-Chandra. The characters of semisimple Lie groups. Trans. Amer. Math. Soc., 83:98-163, 1956.

[8] Henryk Hecht. The characters of some representations of HarishChandra. Math. Ann., 219(3):213-226, 1976.

[9] Henryk Hecht and Wilfried Schmid. A proof of Blattner's conjecture. Invent. Math., 31(2):129-154, 1975.

[10] Henryk Hecht and Wilfried Schmid. Characters, asymptotics and $\mathfrak{n}$ homology of Harish-Chandra modules. Acta Math., 151(1-2):49-151, 1983.

[11] Rebecca A. Herb. Discrete series characters and Fourier inversion on semisimple real Lie groups. Trans. Amer. Math. Soc., 277(1):241-262, 1983. 
[12] Rebecca A. Herb. Two-structures and discrete series character formulas. In The mathematical legacy of Harish-Chandra (Baltimore, MD, 1998), pages 285-319. Amer. Math. Soc., Providence, RI, 2000.

[13] Takeshi Hirai. Invariant eigendistributions of Laplace operators on real simple Lie groups. IV. Explicit forms of the characters of discrete series representations for $\operatorname{Sp}(n, \mathbf{R})$. Japan. J. Math. (N.S.), 3(1):1-48, 1977.

[14] Takeshi Hirai. The characters of the discrete series for semisimple Lie groups. J. Math. Kyoto Univ., 21(3):417-500, 1981.

[15] Anthony Knapp. Representation Theory of Semisimple Groups. An overview based on. Princeton University Press, Princeton, NJ, 1986.

[16] Susan Martens. The characters of the holomorphic discrete series. Proc. Nat. Acad. Sci. U.S.A., 72(9):3275-3276, 1975.

[17] Wilfried Schmid. On the characters of the discrete series. The Hermitian symmetric case. Invent. Math., 30(1):47-144, 1975.

[18] Wilfried Schmid. Some remarks about the discrete series characters of $\operatorname{Sp}(n, \mathbf{R})$. In Non-commutative harmonic analysis (Actes Colloq., Marseille-Luminy, 1974), pages 172-194. Lecture Notes in Math., Vol. 466. Springer, Berlin, 1975.

[19] Jorge Vargas. A character formula for the discrete series of a semisimple Lie group. Bull. Amer. Math. Soc. (N.S.), 2(3):465-467, 1980.

[20] David A. Vogan, Jr. Complex geometry and representations of reductive groups. preprint.

[21] David A. Vogan, Jr. Representations of Real Reductive Lie Groups, volume 15 of Progress in mathematics. Birkhäuser, Boston, 1981.

[22] David A. Vogan, Jr. Irreducible characters of semisimple Lie groups IV. character-multiplicity duality. Duke Math. J., 49, No. 4:943-1073, 1982.

[23] David A. Vogan, Jr. The Kazhdan-Lusztig conjecture for real reductive groups. In Representation theory of reductive groups (Park City, Utah, 1982), volume 40 of Progr. Math., pages 223-264. Birkhäuser Boston, Boston, MA, 1983. 